\title{
Produire une cause victimaire dans un espace de post-conflit : le traitement de la disparition au sein de la communauté turco-chypriote
}

Comment ont-ils disparu?

To create a victimary posture in a post-conflict country: the treatment of disappearance in the Turkish Cypriot community

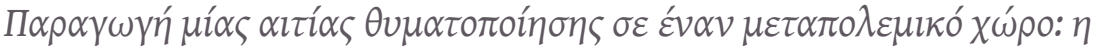

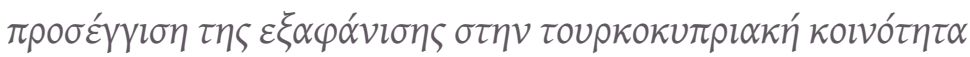

\section{Théotime Chabre}

\section{OpenEdition} Journals

Édition électronique

URL : https://journals.openedition.org/ceb/13791

DOI : 10.4000/ceb.13791

ISSN : 2261-4184

Éditeur

INALCO

Édition imprimée

ISBN : 9782858313341

ISSN : 0290-7402

Référence électronique

Théotime Chabre, «Produire une cause victimaire dans un espace de post-conflit : le traitement de la disparition au sein de la communauté turco-chypriote », Cahiers balkaniques [En ligne], 46 | 2020, mis en ligne le 25 février 2020, consulté le 06 juillet 2021. URL : http://journals.openedition.org/ceb/13791 ; DOI : https://doi.org/10.4000/ceb.13791

\section{(c) (†) 8}

Cahiers balkaniques est mis à disposition selon les termes de la Licence Creative Commons Attribution - Pas d'Utilisation Commerciale 4.0 International. 


\title{
Produire une cause victimaire dans un espace de post-conflit : le traitement de la disparition au sein de la communauté turco-chypriote Comment ont-ils disparu?
}

To create a victimary posture in a post-conflict country: the treatment of disappearance in the Turkish Cypriot community

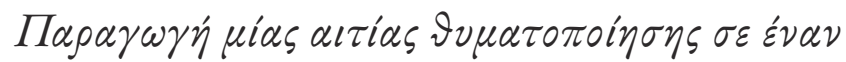

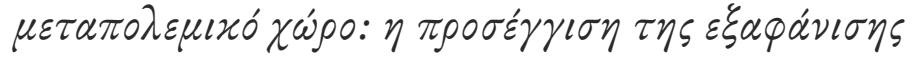

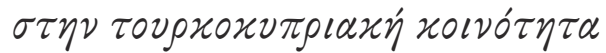

\author{
Théotime Chabre \\ Doctorant, Sciences-Po
}

Le 13 juillet 2007, 300 personnes sont rassemblées au cimetière des martyrs de Nicosie Nord pour assister à un enterrement qui sort de l'ordinaire. Les 13 soldats qui sont enterrés ce jour-là sont morts en 1974, exécutés par l'armée gréco-chypriote pendant l'opération militaire turque qui a abouti à la division de l'ile. Comme 492 Turcs-Chypriotes, ils sont considérés comme disparus pendant la période de guerre civile qui a frappé l'île entre 1963 et 1974. Quelques jours avant, le 8 juillet 2007, les premiers des 1510 Grecs-Chypriotes reconnus comme disparus étaient également enterrés en grande pompe, marquant le début d'une nouvelle ère dans la gestion de la question de la disparition. Car, si un comité représentatif des deux communautés existait depuis 1981 pour localiser, identifier et réinhumer les corps des disparus de l'île, le Comité pour les personnes disparues à Chypre (CMP), pendant les quatre décennies qui ont suivi le conflit, ces disparitions n'ont fait l'objet d'aucune mobilisation ni d'aucun travail d'envergure visant à les localiser. 
Le phénomène est particulièrement visible au sein de la communauté turcochypriote. Si la localisation des disparus a été accaparée par le discours officiel des autorités grecques chypriotes qui en ont fait une cause nationale, la notion même des disparus comme catégorie victimaire distincte est absence du débat politique de la communauté turco-chypriote jusqu'au début des années 2000. Par la suite, particulièrement après l'échec du référendum sur le plan de réunification proposé par le Secrétaire général de l'ONU Koffi Annan en 2004, les disparus prennent une place de plus en plus visible dans le paysage politique turco-chypriote. Des associations se saisissent du sujet et en font une «cause », les administrations publiques investissent dans cette cause en créant des espaces spécifiques dédiés, et la coopération avec le CMP est mise en avant par les représentants politiques de premier plan, relayés par les médias locaux. Ce changement de paradigme au niveau politique transforme également l'investissement des proches pris en compte par le CMP. Dans un second temps, nous identifierons les dynamiques qui ont inhibé la constitution d'une cause victimaire avant les années 2000 puis qui l'ont favorisée dans la première décennie de ces mêmes années. Enfin, nous examinerons les dynamiques actuelles de cette cause en nous appuyant sur les travaux de sociologie portant sur les causes victimaires, notamment les travaux de Sandrine Lefranc et de Delphine Lecombe. Dans le cadre de cette analyse, nous privilégions une approche mixte, nous appuyant sur un corpus historique constitué d'archives et de sources secondaires, et d'une entrée par les acteurs, à travers un travail d'observation au sein des différents groupes au cours d'une enquête de terrain entre janvier et mars 2016.

S'il ne semble pas y avoir eu de conflits communautaires majeurs à Chypre au cours de l'époque ottomane, la montée en popularité de projets nationalistes concurrents au sein de chaque communauté, l'enosis et la taksim, pendant la colonisation anglaise a favorisé des conflits directs et meurtriers entre milices issues des deux communautés principales, les Grecs-Chypriotes et les Turcs-Chypriotes.

\section{Retour sur le conflit intercommunautaire}

En 1958, un premier conflit meurtrier oppose les deux milices, l'EOKA, Ethniki Organosis Kyprion Agoniston (Organisation nationale des combattants chypriotes), et la TMT, Türk Mukavemet Teşkilatı (Organisation turque de résistance), près du village de Gönyeli. Les forces britanniques s'interposent et mettent en place les premières barricades dans la capitale pour isoler les quartiers à majorité turque. En 1960, les Britanniques se retirent de l'île. Un accord a été conclu avec la Grèce et la Turquie, sans consultation des habitants de l'île, pour que Chypre accède à l'indépendance sous la forme d'une république consociationaliste, au sein 
de laquelle le pouvoir est partagé entre les communautés. Mais ce système, peu propice à la constitution d'une identité commune, s'effondre en 1963. Une crise institutionnelle débouche sur le retrait ${ }^{1}$ des représentants turcs-chypriotes au sein de l'État central. À la fin de l'année 1963, des attaques consécutives très violentes ont lieu contre différentes zones d'habitations turco-chypriotes ${ }^{2}$ et marquent le début d'une période de violence intense qui durera jusqu'en juin 1964. Une partie de la population turco-chypriote quitte ses lieux d'origine pour rejoindre des «zones purifiées » balisées par la TMT. Les autorités turco-chypriotes dressent une administration parallèle sur ces quarante-cinq enclaves, «l'Administration temporaire turque » qui correspondent à environ $1,5 \%$ du territoire de l'île. C'est à partir de cette période, et jusqu'à l'invasion militaire turque de 1974, qu'ont lieu les cas de disparitions qui sont actuellement reconnus par les différents acteurs de la cause des disparus à Chypre.

\section{Retour sur les disparitions}

On distingue notamment deux grandes périodes de disparitions, qui recoupent les deux périodes les plus intenses de la guerre civile: la période de trouble de 1963-1964, et l'invasion turque de 1974. À partir de la fin décembre 1963, au milieu des assassinats de civils et des combats entre milices, des enlèvements massifs sont organisés au sein des deux communautés. Les Turcs-Chypriotes sont les plus touchés par cette pratique, et plusieurs centaines d'entre eux sont enlevés entre le 21 décembre 1963 et juin 1964³. Le CICR, appelé par les Britanniques en

1. La question de savoir si les représentants turcs-chypriotes ont été expulsés par les leaders politiques grecs-chypriotes ou s'ils sont partis sous pression des milices armées turques chypriotes n'est pas tranchée.

2. Certains soutiennent que les exactions sont le fait d'anciens membres de l'EOKA, dissoute officiellement en 1959, que le gouvernement ne contrôle pas. D'autres penchent plutôt pour un plan coordonné avec les autorités, le plan Akritas, dont le but est de plonger les Turcs-Chypriotes dans un climat de peur à même de les pousser à quitter l'île. 3. La plupart des disparitions semblent s'inscrire dans des enjeux locaux de contrôle de la terre, notamment dans les régions rurales et en périphérie des zones mixtes. Le cœur des zones d'habitation mixtes, notamment les plus grands centres urbains, semble avoir été plutôt épargné. Un élément d'explication, souvent relevé par les enquêtés, pourrait être la force des réseaux d'interconnaissances, renforcés par des activités syndicales transcommunautaires. Ces pratiques systématiques de l'enlèvement ne semblent pas relever d'un plan coordonné des autorités. Mais, bien qu'elles s'inscrivent le plus souvent dans des évènements locaux, la temporalité, les méthodes d'enlèvement et la constitution d'un réseau de caches destinées à «entreposer» une partie des enlevés suggèrent une forme de coordination avancée. En d'autres mots, si la responsabilité directe de 
58 Politique et sociétés à Chypre aujourd'hui

réponse aux violences, met en place des campagnes de recherche et dresse des listes de victimes qui distinguent pour la première fois une catégorie « disparus » pour ceux dont il n'a pas été possible de déterminer le destin. Le bilan de cette période s'élève, selon le Secrétaire général de l'ONU de l'époque, U Thant, à 208 disparus turcs-chypriotes et 38 disparus grecs-chypriotes ${ }^{4}$. Entre 1964 et 1974, le statu quo domine les relations entre les deux communautés. Les cantons sont mis sous embargo par la République de Chypre, renforçant les difficultés économiques des Turcs-Chypriotes. Les confrontations directes sont épisodiques, seuls neuf cas de disparition sont notifiés en 1965 et 1967, et aucun entre 1968 et 1972.

Le coup d'État des partisans de l'EOKA-B contre Makarios le 15 juillet 1974, met à bas cet équilibre précaire. Le 20 juillet suivant, la Turquie lance en réaction une opération militaire, baptisée «force de paix» pour « restaurer l'ordre constitutionnel » au terme de laquelle elle contrôle 38 \% de l'île. Malgré le retour de Makarios au pouvoir elle ne retire pas ses troupes, et le fait accompli est entériné en août 1975 avec les derniers échanges de populations et l'installation de l'Autorité temporaire turque dans les territoires occupés. Pendant les mois de juillet et août 1974, les exactions concernent principalement les Grecs-Chypriotes, mais des massacres de Turcs-Chypriotes ont également lieu. Plusieurs milliers de combattants de tout bord sont tués ou blessés. Concernant les disparitions, le CICR, qui renvoie une équipe sur place dès juillet 1974, recense plus de 1614 soldats grecs-chypriotes disparus. Trois massacres de masses de TurcsChypriotes ont lieu pendant la retraite des soldats grecs, entre le 14 et le 15 août ${ }^{5}$ et on compte parmi eux 84 disparitions.

À partir de ces évènements, des récits mémoriels concurrentiels se développent au sein des deux communautés. Chacune d'elles désormais séparée géographiquement et structurée autour d'un appareil étatique distinct, développe une mémoire tronquée, centrée sur ses propres victimes, occultant les victimes de

l'administration grecque-chypriote est discutée par l'anthropologue Sant Cassia, l'aspect organisé et systématique de l'enlèvement est clair.

4. Sur la même période, 38 Grecs-Chypriotes enlevés ne sont pas revenus et 174 ont perdu la vie. En ce qui concerne les autres victimes, 364 Turcs-Chypriotes et 174 GrecsChypriotes perdirent la vie. 18667 Turcs-Chypriotes durent fuir leur domicile. CAssia, 2004.

5. Dans les trois villages de Muratağa, Sandallar et Atlılar, 126 femmes, enfants et personnes âgées sont assassinées en l'absence des hommes du village, faits prisonniers le 20 juillet. Dans les villages de Mari, Zigi et Dochni, les 84 hommes du village sont enlevés et exécutés dans les jours qui suivent. À Aleminyo, dans le territoire sous contrôle des Grecs-Chypriotes, 13 soldats prisonniers sont exécutés et enterrés dans une fosse commune. 
l'autre côté. En parallèle, un dialogue se met en place entre les différents acteurs du conflit autour de la perspective de réconciliation. La thématique de la disparition va rapidement devenir un enjeu au sein de ces négociations qui vont donner naissance au CMP.

Il convient d'analyser ces deux processus, l'un qui contribue à développer un cadre mémoriel communautaire et l'autre, qui émerge comme objectif de négociations dans le cadre des discussions entre les dirigeants engagés dans le processus de réunification. Les deux vont produire des visions très différentes. L'interaction des cadres mémoriels avec les modalités des politiques de postconflit mises en place par les communautés permet de mettre en lumière des mécanismes qui nous éclairent sur l'absence de revendications victimaires visibles jusqu’au début des années 2000.

\section{Faire disparaître la disparition : 1968-2002}

\section{La construction du culte des martyrs turco-chypriotes}

Au sein de la communauté gréco-chypriote, le récit mémoriel évacue rapidement les violences de 1963-1964 pour se concentrer sur celles liées à l'invasion turque de 1974. La perte d'un tiers du territoire de l'île, le décès de milliers de compatriotes, l'arrivée massive de déplacés dans les territoires qui sont restés sous contrôle de la communauté et l'échange de populations qui fait quasi totalement disparaître toute cohabitation avec la communauté turco-chypriote contribuent à faire de l'invasion militaire turque une catastrophe nationale, fondatrice de la nation grecque-chypriote moderne. Dans cette mémoire du conflit, la place des disparus sera centrale. Progressivement, les Agnooumeni («disparus») sont sanctifiés religieusement et reconnus politiquement comme un symbole de la permanence de l'oppression turque et du martyr de Chypre ${ }^{6}$. Ils deviennent des figures de l'oppression turque: le nombre de disparus identifié par le CICR, 1614, devient un symbole connu de tous les écoliers, des monuments religieux et civils sont érigés en leur honneur. Pour les autorités, ils sont des prisonniers, vivants, mais prisonniers dans un lieu tenu secret par la Turquie, et leur captivité devient un symbole de la permanence de l'occupation. La place que prennent les disparus dans le discours nationaliste devient telle qu'il est désormais quasiment

6. La République de Chypre se retrouve amputée de $38 \%$ de son territoire et doit gérer l'intégration de plus de 200000 réfugiés, pour un coût annuel estimé à 77 millions de dollars pendant la seconde moitié des années 1970 selon un rapport d'État américain. Special report on Cyprus released by senator Kennedy, 21 juin 1976, Wikileaks. 
impossible de réviser ces cas à l'aune de nouvelles informations au cours des années qui suivent ${ }^{7}$.

Au sein de la communauté turco-chypriote, le scénario est tout autre. En 1974, la disparition est déjà un «dossier bouclé » pour les autorités ${ }^{8}$. Elles ont pris le parti de considérer légalement les disparus comme morts dès 1968. Pour elles, la disparition en elle-même ne constitue pas une «exaction spécifique ». Elle fait partie d'un plan coordonné de nettoyage ethnique, le «plan Akritas », qui aurait visé à pousser au départ les Turcs-Chypriotes de l'île pour faciliter la mise en place de l'enosis ${ }^{9}$. Par ailleurs, contrairement aux Grecs-Chypriotes, les autorités turques-chypriotes promeuvent un cadre mémoriel qui considère une période de violence beaucoup plus large, de 1955 à 1974, marquée par deux évènements majeurs, décembre 1963 et juin 1974.

Le souvenir de la semaine de violence de la fin décembre 1963 devient un des deux «socles mémoriels» sur lesquels se fixe la mémoire des conflits communautaires. Elle entre dans l'histoire comme Kanlı Noel, le «Noël sanglant ${ }^{10} \gg$. Entre 1963 et 1974, une entreprise de propagande d'État est mise en place pour montrer au monde l'injustice faite aux Turcs-Chypriotes, dans le cadre des luttes diplomatiques pour obtenir une légitimité face à la communauté internationale. Cette propagande est fondée sur une monstration de la violence en se concentrant sur des photographies d'époque, mais également sur la sacralisation

7. Une enquête du journaliste Andreas Paraschos a notamment montré comment une centaine de soldats considérés comme disparus étaient enterrés, au su des autorités, dans le cimetière de Nicosie, en territoire contrôlé par les Grecs-Chypriotes.

8. La mention des «autorités turques-chypriotes» ou de la «République turque de Chypre du Nord» ne signifie pas une reconnaissance de la part de l'auteur de l'État autoproclamé dans les zones occupées par la Turquie.

9. L'existence historique du plan Akritas est attestée par la plupart des historiens ayant travaillé sur Chypre, mais ils sont plusieurs à souligner, comme Sant Cassia, que les autorités officielles ne semblent pas avoir pu exercer un véritable contrôle sur le déroulement des évènements. Ainsi, Mauss-Copeaux et Copeaux présentent l'hypothèse du plan Akritas parmi d'autres et Sant Cassia s'appuie sur les journaux de l'époque pour décrire une situation «beaucoup moins gérable qu'aucun plan aurait pu envisager, anticiper ou même en profiter » (CASsia, 2004, p. 26). L'hypothèse historique que constitue le plan Akritas est en tout cas érigée par les autorités turco-chypriotes et turques en fait tangible qui justifie, a posteriori, la construction d'une administration parallèle dans les cantons.

10. C'est un qualificatif qui pourrait avoir été d'abord popularisé par les correspondants de presse européens qui rapportent les évènements. Pour une utilisation contemporaine de la référence historique de Kanlı Noel dans les milieux nationalistes turco-chypriotes, on peut consulter le numéro spécial de Direniş, la revue de l'Association des Mücahit (combattants) de Chypre, du 21 décembre 2015. 
des lieux où furent commis les massacres, comme le montre l'exemple du musée de la Barbarie. Dans ce cadre, la disparition, forcément invisible, est tenue à l'écart de la propagande officielle. Ce répertoire de monstration est renforcé après le conflit de 1974, notamment en raison du grand nombre de victimes turques-chypriotes dont le sort est connu et dont les cas ne sont pas, à proprement parler, des cas de disparition. À Muratağa, Sandallar et Atlılar, lieux du plus grand massacre de Turcs-Chypriotes pendant le conflit, les victimes sont découvertes quelques jours plus tard, par les forces armées turques. Dans le cas de Mari, Zigi et Dochni, lieu d'un autre massacre dont les victimes n'ont pas été retrouvées, la nouvelle de leur exécution est divulguée rapidement par un survivant ${ }^{11}$. Les récits de l'ouverture des fosses en 1974, publiés dans la presse et dans les rapports de l'ONU, remplacent progressivement Kanlı Noel comme preuve numéro 1 du $\left\langle\right.$ sacrifice ${ }^{12} \gg$ des TurcsChypriotes. Le récit officiel, qui semble partagé par une partie importante de la population et qui fait du massacre de Muratağa-Sandallar-Atlılar l'image de l'enfer, est apocalyptique: sans l'intervention armée de la Turquie, la seule issue de la communauté aurait été la disparition ${ }^{13}$. À cette image, on renvoie l'image de la victoire, qui voit la communauté, quelques mois auparavant en situation défavorable, disposer désormais de la protection de l'armée turque et d'un nouveau territoire vingt fois plus grand que celui des enclaves.

Les disparus, majoritairement présentés comme des victimes, n'ont pas leur place dans ce discours victorieux. Cette construction propagandiste est à la fois destinée à présenter aux partenaires internationaux les revendications turqueschypriotes sous un jour favorable et à rassembler une communauté divisée vis-à-vis du projet sécessionniste. L'effacement de la question de la disparition permet à la fois de ne pas insister sur l'époque pendant laquelle la communauté vivait sous domination et de ne pas couper tous liens avec les territoires abandonnés.

11. Pour une mise en récit de l'enlèvement des hommes de Mari Zigi et Dochni, on peut se référer à ULUDAĞ Sevgül, 7 juillet 2013.

12. Il existe deux mots en turc pour parler de victime: mağdur et kurban. Alors que mağdur a le sens «neutre » et descriptif du mot anglais «casualty», kurban a une portée plus «morale». Il porte à la fois la connotation d'empathie, que le mot français «victime » véhicule, et celle de sacrifice dans l'eschatologie musulmane, c'est-à-dire, ce qui permet d'atteindre un but, dans le sens de la phrase «son sacrifice n'a pas été vain. » Â noter qu'à la différence de la notion de sacrifice dans la tradition chrétienne, il n'y a pas l'idée d'expiation à travers l'acte sacrificiel dans la tradition musulmane.

13. Voir l'ouvrage produit par l'Association de commémoration des martyrs de MuratağaAtlılar-Sandallar : 1955-1974 step by step Genocide rédigé en 2007 qui reprend une partie des codes de publicisation victimaire des autorités turco-chypriotes. 
Au-delà de ce discours, la mise de côté des proches de disparus se renforce à travers la mise en place d'un système de redistribution des ressources matérielles et symboliques issues de l'invasion turque, un des éléments fondateurs de l'administration du nouvel «État» en construction dans le nord de Chypre. Ce système permet de légitimer le contrôle des ressources et de l'État par la faction politico-militaire qui succède au groupe de résistance turco-chypriote, les Mücahit (combattants) de la TMT ${ }^{14}$. Il est construit autour d'un culte des martyrs local inséré dans le culte des martyrs de la République de Turquie, qui veut renforcer la «turcité » de la communauté et pose les Mücahit comme gardiens de cette turcité. Par ailleurs, ce culte des martyrs s'appuie sur une classification des mérites des victimes, qui porte au sommet les victimes combattantes de la TMT, qu'ils soient Sehit (martyrs) ou blessés (Malül Gazi). Ce culte pose les cas des disparus comme des cas limites, les rejetant dans les limbes de la reconnaissance sociale ${ }^{15}$.

\section{Lutte pour le contrôle des ressources et mise en place de cas limites}

Au cours de l'année 1974, la loi relative à l'aide donnée aux familles des martyrs et victimes des évènements ainsi qu'aux invalides est votée par les autorités turco-chypriotes ${ }^{16}$. Elle ouvre la possibilité aux victimes du conflit de demander à bénéficier d'une aide d'État. Le texte définit les catégories victimaires et les compensations auxquelles elles ont droit. La validation des dossiers est sous la responsabilité d'un comité d'évaluation dont les membres sont en partie nommés parmi les forces armées. La loi couvre un large spectre d'aide: des pensions mensuelles sont octroyées aux invalides, aux mères et femmes des victimes. Les pères et les enfants des victimes peuvent recevoir un terrain ou, dans certains cas, une propriété, issues des prises de guerre. L'ensemble des personnes concernées bénéficie de facilités pour obtenir un emploi public et des bourses d'études.

À travers cette loi, deux catégories de victimes sont dissociées. D'un côté les invalides de guerre et leurs conjoints et enfants ainsi que les proches de martyrs, et de l'autre les proches et invalides des «victimes des évènements» (Hadise Kurbani ${ }^{17}$. La catégorie des martyrs comprend exclusivement ceux décédés dans l'exercice de leur fonction officielle ou officieuse de mücahit. Le statut de victime

14. L'ancien combattant, ou mühacit, est défini comme le personnel combattant engagé au sein des forces armées chypriotes turques (Loi 7/1974 - Aides aux familles des martyrs et des vétérans de guerre, s. d.).

15. D'autres cas limites sont identifiés, comme les individus assassinés.

16. Loi 7/1974 - Aides aux familles des martyrs et des vétérans de guerre.

17. Les martyrs et les «victimes des évènements » sont définis comme ceux ayant perdu la vie ou ayant disparu au combat ou à l'entrainement entre le $1^{\text {er }}$ avril 1955 et le 31 décembre 1958 et du 21 décembre 1963 à aujourd'hui. À l'exception de quelques cas 
des évènements correspond officiellement aux civils. Dans les faits, ce statut a été assigné à une série de profils «marginaux » qui ne pouvaient être clairement identifiés comme militaires. Les victimes d'enlèvements et d'exactions entre 1963 et 1964 et les victimes civiles de massacres en 1974 relèvent ainsi de ce statut. D'autres cas limites sont identifiés, comme les individus assassinés par la TMT pour avoir dénoncé le projet sécessionniste ${ }^{18}$.

Les disparus ne font pas l'objet d'une catégorie spécifique, mais la loi classe les militaires disparus comme martyrs, si la cause de leur disparition apparaît clairement comme dépendante d'une action ennemie. Dans les faits, l'impossibilité de prouver la disparition a justifié l'intégration de l'ensemble des cas de disparus à la catégorie de «victime des évènements », instillant un soupçon vis-à-vis du «mérite » à attribuer à ces victimes. Le disparu est un cas qui peut relever facilement de ce que Lefranc et Mathieu appellent la victime douteuse, « exposée au soupçon de présenter des attentes de reconnaissance et de réparation indues ${ }^{19} \gg$. L'absence de preuve de sa mort favorise la diffusion d'hypothèses, de rumeurs, qui n'ont pas besoin d'être irréfutables ni même plausibles pour instiller le doute. Mais ce doute est explicatif dans une moindre mesure. En effet, si le sort final de la victime est inconnu, le fait qu'il ait été enlevé est le plus souvent attesté. Remettre en question les témoignages à ce point-là semble plutôt relever d'une volonté de la part des autorités compétentes de décrédibiliser certains profils pour influencer le découpage des ressources issues de la redistribution des «prises de guerre » à travers le système de compensation. Ce sont autant de profils dont les failles sont exploitées pour établir une «mesure du sacrifice» qui permet de légitimer la différenciation des compensations.

Pour les proches de disparus, ce traitement différencié a deux conséquences directes: financière d'abord, puisque la pension que touche la famille est réduite de moitié par rapport aux enfants et aux femmes de martyrs ${ }^{20}$, et symbolique, les disparus ne bénéficiant ni des cérémonies militaires ni de l'enterrement dans les carrés réservés aux martyrs. La mise en place de ces distinctions permet également par la suite de soutenir la création d'un système de points visant à évaluer l'attribution des propriétés abandonnées qui favorise les mücahit, les invalides de

de morts en entraînement dans les années 1980, aucune perte n'est signalée après la fin de l'opération militaire turque.

18. Hatay, 2016.

19. Lefranc \& Mathieu, 2009.

20. Les pensions accordées témoignent de la hiérarchisation. Les blessés de guerre reçoivent la plus forte pension, entre 2200 et 5000 lira par mois selon le handicap. Les femmes de martyrs reçoivent 3200 lira par mois. Jusqu'en 2002, les femmes de « victime des évènements » reçoivent environ moitié moins que les femmes de martyrs. 
guerre et les familles de martyrs, prioritaires face aux déplacés et aux proches des « victimes des évènements ${ }^{21}$ ».

Cette classification administrative ne rencontre aucune opposition notable jusqu'en 2002. La pérennisation du système, malgré son injustice certaine, peut s'expliquer en partie par le contrôle exercé par le régime de Rauf Denktaş, issu des mücahit, sur la vie sociale, et par l'allégeance envers le régime de l'association qui représente les intérêts des victimes du conflit. Cette association soutenue par le gouvernement et dominée par les anciens combattants, l'Association des familles de martyrs et des invalides de guerre ${ }^{22}$, revendique représenter $65 \%$ des familles de la communauté, ce qui contribue à diluer les cas de proches de disparus dans une plus large masse ${ }^{23}$.

L'accentuation par la catégorisation administrative d'un ordre moral qui distingue le combattant actif des autres victimes participe ainsi à un ensemble de lutte au sein du nouvel État pour le contrôle des ressources matérielles et symboliques. En prenant appui sur une définition du martyr qu'ils ont adaptée à leur image, les mücahit, à travers leur domination de l'administration et du gouvernement turco-chypriote, participent à institutionnaliser l'appropriation des «biens de conquête » et leur domination sur l'appareil étatique turco-chypriote.

\section{L'absence de mobilisation des proches de disparus}

Ces éléments ne permettent pas toutefois d'expliquer pourquoi aucun mouvement d'activistes ne s'est développé à l'époque autour de revendications propres à la disparition. Dans d'autres cas de disparitions massives étudiées

21. "Only in 1977 was a points system established that attempted to assign properties on the basis of what Turkish Cypriots had left behind in the south. The system, however, was reportedly rife with nepotism, favoritism, and various forms of discrimination, including for political reasons. Şehit aileleri, or persons who had lost loved ones in the conflict, received a house and small amount of monthly compensation. In addition, after 1989, men who had been mücahits, or fighters, during the 1958-74 period received points that could be used towards acquisition of property" (BRYANT, 2012).

22. Kıbrıs Şehit Aileleri ve Malül Gaziler Derneği.

23. L'association est ouverte à toutes les victimes du conflit: les invalides de guerre, les parents, conjoints et enfants de martyrs. L'association ne mentionne aucune autre catégorie, ni «victime des évènements » ni « disparus », mais la mention de martyr est à prendre ici au sens large. En 2016, l'association compte officiellement 7000 membres, sur 40000 membres potentiels. Elle reconnaît 1500 familles de martyrs, soit $65 \%$ des familles de l'île. Parmi elles, 500 familles relèveront du statut de Hadise Kurbani jusqu'en 2002. 
précédemment, notamment au Chili ${ }^{24}$, l'opposition de l'État n'empêche pas la mobilisation des proches, mais, au contraire, participe à souder un mouvement autour de la dénonciation du traitement qui leur est réservé. Des différences structurelles peuvent ici être mises en avant et ressortent de l'analyse des profils et des trajectoires des proches de disparus. Une première piste constitue le fait qu'à la différence des disparitions dans plusieurs pays d'Amérique du Sud, l'État qui est susceptible d'être identifié comme coupable des crimes, ou les criminels eux-mêmes, font partie d'une société qui est géographiquement et politiquement distincte après 1974. L'État turco-chypriote, s'il peut être tenu responsable du traitement réservé aux proches des victimes, n'est pas tenu comme directement responsable des disparitions.

La publicisation d'une cause victimaire, comme pour n'importe quelle cause avant l'arrivée d'internet, demande l'accès à des interfaces de communication, manifestations sur la place publique, mobilisations auprès des administrations compétentes, avec les pouvoirs publics accusés d'être responsables de l'exaction concernée. Pour les proches des victimes, il est physiquement impossible de saisir la justice dont relèvent les présumés coupables. Les traces de manifestations de proches de disparus en 1964, avant la séparation définitive, vont dans ce sens. La sous-traitance de la crise aux organisations internationales, puis le blocus international et le contrôle strict du débat public au sein de l'«État turcchypriote » ne font que renforcer l'impossibilité de formulation d'un problème commun et donc la constitution d'une communauté de destin ${ }^{25}$. Il est frappant de constater que les manifestations de 1964 n'ont par ailleurs pas donné lieu à une mémoire collective et qu'aucun témoignage n’a renvoyé spontanément à ces cas pourtant dûment documentés.

À l'échelle individuelle, l'analyse des profils socioéconomiques des proches de disparus ainsi que leurs trajectoires avant, pendant et après le conflit offrent des perspectives intéressantes pour mieux comprendre ce phénomène. Des proches donnent des informations quant à leur incapacité à transformer leurs souffrances personnelles en une «substance éthique collective $^{26}$ puis en revendications communes.

En effet, ils sont pour la plupart des gens issus de familles faiblement dotées en capital économique, plus susceptibles d'avoir vécu un ou deux exils au cours des années 1960 et 1970 et d'avoir dû vivre plusieurs années dans des camps

24. Diaz \& Gutiérrez Ruiz, 2009.

25. Ibid.

26. Ibid. 
de déplacés. La plupart sont des femmes, dans une société où le taux d'emploi des femmes était beaucoup moins important que celui des hommes. Parfois accompagnées d'enfants à charge, elles doivent subvenir à leurs besoins dans une société et à une époque où la condition féminine est peu propice au travail, ce qui les rend d'autant plus dépendantes de l'aide de l'État. 246 des 376 disparus identifiés, soit plus de deux tiers à avoir disparu, avaient entre 20 à 40 ans, ce qui laisse supposer que les cas de femmes seules avec enfants à charge constituent une grande partie des cas de conjoints de disparus. À cet état de fait, il faut ajouter les violences invisibles, notamment les nombreux cas de viols qui ont été commis pendant les violences, et ce potentiellement sur des femmes de disparus. Ces différents facteurs ont entraîné parmi elles une grande détresse économique, les privant des ressources nécessaires à l'action collective, comme le temps, la capacité de déplacement et une autonomie financière minimale vis-à-vis de l'État.

L'absence de mobilisations qui répondent aux critères «traditionnels » de la sociologie des mouvements sociaux ne signifie pas pour autant que la disparition n'entraîne pas une série de résistances quotidiennes ${ }^{27}$ face à l'impératif d'oubli, sous la forme de comportements propres à maintenir l'attente du disparu, comme le suivi des rumeurs, qui évoquent une nouvelle vie ailleurs pour leurs proches, le recours à des services de voyance ou le refus de réaliser les prières de souvenirs aux morts. Ces formes de résistances quotidiennes représentent autant de marques de refus d'un cadre mémoriel imposé par le haut.

Par ailleurs, il faut noter une différence importante entre les cas de familles victimes de la disparition individualisée d'un de leurs proches, notamment les cas de «disparus sur la route» et les familles de disparus faisant partie d'une disparition massive, qui a concerné une localité précise, comme à Aleminyo, ou un entier groupe de travailleurs, comme le cas du Kayzp Otobus (l'autobus disparu). Ces groupes, déjà liés par des liens familiaux ou de voisinage, ont pour la plupart connu un déplacement collectif dans un village du Nord, ce qui a permis le maintien d'une cohésion et un partage du souvenir.

\section{Une «problématique de la disparition» affichée dans le cadre des négociations}

Si, de 1974 au début des années 2000, se mettent en place des politiques mémorielles internes à chaque communauté, sans perspective de collaboration,

27. Ce développement reprend certains concepts développés par BAYAT, 2010. Comme le «pauvre » de Bayat, ils se retrouvent marginalisés, symboliquement exploités par une administration qui puise dans leur récit une ressource morale, mais les confine à une catégorie administrative subalterne. Stigmatisés socialement en tant que «victime» passive des évènements, ils sont exclus d'un système politique qui valorise le combattant. 
la situation est tout autre au sein des négociations qu'organise la coalition internationale qui sponsorise les négociations entre les deux communautés. Ces négociations sont menées par des acteurs qui n’ont pas les mêmes agendas, mais sont publicisées comme un effort engagé en vue d'un rapprochement et de la formulation d'une solution de compromis qui permettent de réunifier l'île. Dès le début, devant la difficulté de la tâche et les intérêts contradictoires des participants, des projets annexes, moins coûteux politiquement, ont été soumis à discussion dès la fin de la guerre. La mise en place d'un organisme commun de recherche des disparus, appuyé par l'ONU, en constitue un des projets les plus aboutis.

La création du Comité pour les personnes disparues de Chypre (CMP) en 1981 est souvent décrite dans les discours officiels comme le produit d'une volonté de travail commun, exemple toujours inédit à l'heure actuelle d'une institution associant des représentants publics des deux communautés. Elle est également le résultat d'un processus de négociation au sein duquel les belligérants cherchent à protéger leurs intérêts et à ne jamais apparaître sous un moins bon jour que leur opposant. Si les représentants grecs-chypriotes ont défendu cette commission dès 1975, dans le but de forcer la Turquie à coopérer pour faire la lumière sur les disparition ${ }^{28}$, les autorités turco-chypriotes déclarent dans ce cadre avoir également des disparus et qu'un tel comité devrait s'occuper des cas des deux communautés. En 1978, après des négociations difficiles et l'accord officiel de la Turquie, dont les autorités turco-chypriotes dépendent pour leur politique étrangère ${ }^{29}$, les deux parties s'accordent pour la mise en place d'un comité bilatéral. En 1981, le Comité pour les personnes disparues (Committee on Missing Persons) est mis en place avec le soutien de l'Assemblée générale de l'ONU et la participation du CICR. Il est encadré par un mandat en douze points qui définit sa mission comme: «déterminer le sort des disparus ». De 1981 à 1997 il est formé de trois membres, deux représentants des administrations de l'île et un du CICR nommé par l'ONU. Chacun dispose d'un à trois assistants. Pendant cette période, son travail a consisté à dresser des listes officielles.

28. Jusqu'au début des années 2000, les autorités de la République de Chypre ont continuellement affirmé que les disparus grecs-chypriotes étaient captifs en Turquie.

29. La République turque de Chypre du Nord n'est pas reconnue par la communauté internationale. Seule la Turquie lui offre une certaine reconnaissance institutionnelle. Dans les faits, la Turquie gère en premier lieu une grande partie de ses décisions administratives et politiques, à travers ses représentants militaires et le «Comité de soutien » de l'ambassade turque de Nicosie, sorte de gouvernement parallèle qui détient un droit de regard et de véto sur un large panel de domaines. Confidential, 1 September 1978, Public Library of US Diplomacy, 1978. 
Née à une époque où la question de la recherche des disparus fait des remous en Amérique latine, l'initiative a l'avantage de s'appuyer sur des listes dressées par le CICR, de ne pas officiellement devoir servir de base à des poursuites judiciaires et de ne pas inclure dans ses missions la recherche des responsables. Pourtant, le comité semble n'avoir guère été plus qu'une coquille vide entre 1981 et 1997, date à laquelle le représentant de l'ONU demande sa dissolution face aux blocages politiques indépassables provoqués par les deux gouvernements de l'île. De fait, s'il n'est pas ici question de distribuer des points, il existe des blocages des deux côtés, notamment du fait qu'aucun des deux gouvernements ne souhaite faire apparaître publiquement les crimes commis par des membres de sa communauté. Ces blocages sont tels qu'en 16 ans de travail quotidien, le résultat est quasi nul, il n'existe même pas d'accord sur les listes de disparus, et les proches de disparus n’ont jamais été inclus dans le processus.

Ce que nous apprend la naissance du CMP, c'est que si la thématique de la disparition est promue comme un non-sujet par les autorités turco-chypriotes en interne, elle est au cœur de la stratégie de propagande et de négociation de ces autorités face à la République de Chypre et aux acteurs internationaux impliqués dans le conflit. Malgré l'échec apparent du comité, l'expérience se révèle décisive après un rapide bouleversement de la situation politique à Chypre au début des années 2000. Ce bouleversement entraîne en effet par ricochet un réinvestissement global de la question des disparus du CMP.

La crise économique qui frappe le Nord de Chypre via la Turquie à la fin des années 1990 favorise la montée d'un rejet du régime de Denktaş en République turque de Chypre du Nord. Ce rejet prend la forme d'un mouvement de contestation dont le cœur est un ensemble d'associations qui promeuvent la réunification de l'île. Ce phénomène, corrélé à un certain rejet de la relation spéciale qu'entretiennent la Turquie et l'État et à la consolidation de l'opposition sous l'égide du Parti républicain du peuple (CTP), accentue la pression sur le régime qui tente de réformer certaines de ses approches pour maintenir sa base. Deux de ces approches transforment le rapport de l'État avec les proches de disparus. Une des actions principales est l'ouverture unilatérale du mur qui sépare les deux côtés de l'île, donnant pour la première fois depuis près de trois décennies la possibilité aux Chypriotes d'aller de l'autre côté. Cette ouverture permet aux proches de disparus d'entamer de nouvelles recherches et rend possible la mise en relation de proches des deux communautés. Une autre action consiste à donner des gages aux proches de disparus directement, en égalisant les rétributions financières et morales des «victimes des événements » avec celles des martyrs, à travers une demande officielle de l'Association des familles de martyrs le 7 mai 2002, et en appuyant des recours judiciaires auprès de la Cour européenne des Droits de l'homme la même 
année $^{30}$. Si le nombre de proches concernés est limité et si les recours échouent, ils représentent une des méthodes que le régime déploie pour réactualiser sa légitimité en tant que protecteur de la communauté face à l'oppression gréco-chypriote, et pour élargir son soutien populaire. En contrepartie, cet engagement implique une reconnaissance officielle de la disparition, qui rend d'autant plus difficile de ne pas s'engager dans un processus international, malgré de fortes résistances, incluant la réclusion d'informations aux proches de disparus demandeurs et le déplacement de fosses communes avérés jusqu'au début des années $1990^{31}$.

À l'époque, le CMP semble mort et enterré, mais il revient pourtant sur le devant des négociations à peine deux ans plus tard. Un an après, en 2003, le régime de Denktaş est renversé par les urnes et le CTP de Mehmet Ali Talat, favorable à la réunification, arrive au pouvoir ${ }^{32}$. En 2004, après l'échec du référendum sur le plan Annan, les négociations sont rompues entre le gouvernement de Talat et celui de Papadopoulos. Les partenaires internationaux, l'ONU de Koffi Annan en tête, poussent à la relance du CMP pour garantir un canal de communication, cherchant un objectif de coopération plus atteignable. Ce canal de communication est cette fois hautement publicisé et promu comme une institution dépolitisée, à caractère uniquement «humanitaire ». L'objectif principal est l'appui aux victimes et le traitement des blessures liées au conflit. Les procureurs des deux territoires s'engagent à ne pas poursuivre pénalement les personnes qui viendraient divulguer des informations au CMP. Il ne s'agit pas tant de résoudre le crime que de venir en aide aux proches des disparus. C'est la promotion d'une cause victimaire des proches de disparus, au lieu d'une cause de la disparition, qui permet aux autorités de promouvoir ouvertement le nouveau CMP sans devoir

30. Cinq mois après le changement de statut des proches de disparus, les premières plaintes de proches de disparus turco-chypriotes auprès de la Cour européenne des Droits de l'homme $(\mathrm{CEDH})$ sont déposées par des avocats employés par la présidence de la République turque de Chypre du Nord. Baybora et autres c. Chypre (déc.), n 77116/01, 22 octobre 2002 .

31. Le maintien de la disparition, ou la volonté de ne pas avoir à avouer la connaissance des fosses communes expliquent en partie ces activités de vidage de fosses. Mais d'autres considérations interviennent, notamment l'investissement immobilier et les résistances des communautés locales face à la perspective d'une enquête judiciaire (UluDAĞ, 2004).

32. Le 23 avril, le plan est soumis simultanément aux Turcs-Chypriotes et aux GrecsChypriotes par référendum. Du côté des Turcs-Chypriotes, le soutien à l'adhésion s'affiche clairement: le «oui » remporte $64 \%$ des voix. Mais le «non» à $76 \%$ des Grecs-Chypriotes, appelé par les partis au pouvoir, enterre définitivement la perspective de réunification. Le $1^{\text {er }}$ mai suivant, la République de Chypre entre dans l'Union européenne, mais reste divisée. 
70 Politique et sociétés à Chypre aujourd'hui

remettre en cause les cadres mémoriels communautaires ou apparaître comme relançant les négociations.

On peut voir cette dépolitisation se traduire discursivement par la promotion d'un vocabulaire qui désigne les victimes du Sud et du Nord comme "missing persons", des personnes manquantes. Ce terme, au contraire du terme « disparu » ne renvoie pas aux causes de la disparition. La personne n'est tout simplement pas là où elle devrait être et le CMP s'en tient à réparer cette «erreur ». Le label humanitaire apposé aux travaux du CMP, tout comme la structuration de mouvements d'oppositions bicommunautaires et l'engagement de poursuites auprès de la $\mathrm{CEDH}$ creusent néanmoins des brèches dans une mémoire officielle qui avait exclu la reconnaissance de la spécificité de la disparition tout comme la reconnaissance des crimes de guerre. Cette brèche sera par la suite exploitée par un ensemble d'acteurs qui profitent des possibilités offertes par ce nouvel espace pour se saisir de la cause des proches de disparus. C'est le processus de formation et d'évolution du champ de cette cause au cours de cette nouvelle période (2004-2016) qu'il convient maintenant d'analyser, en s'appuyant sur les pratiques des groupes d'acteurs qui vont s'y investir, pour observer comment fonctionne une cause défendue par des acteurs ayant des inscriptions politiques et professionnelles diverses et parfois contradictoires.

\section{La cause de la disparition aujourd'hui}

Pour approcher cette deuxième partie, il nous semble préférable de changer de prisme et d'échelle d'analyse. Contrairement à la première période étudiée où la production des discours et des politiques publiques se fait soit dans l'espace clos de la communauté; soit dans celui tout aussi cloisonné des couloirs et des salles de négociations internationales, la situation est beaucoup plus floue en 2004. La promotion de la cause des proches de disparus se construit dans une île dont le mur s'est ouvert, traversée par des mouvements citoyens qui dépassent les frontières communautaires. Cette cause devient à la fois le laboratoire d'un travail collectif et le cache-misère d'un processus de réunification en berne. Le débat politique s'est ouvert avec la chute du régime de Rauf Denktaş, et les langues se sont déliées, si bien que plusieurs groupes s'investissent dans la question de la disparition, développant des pratiques de coopération par moments et de concurrence à d'autres, avec des objectifs politiques différents. Ces acteurs sont engagés dans un processus de reconfiguration permanente à travers le rapport aux autres mouvements, ce qui pousse Lilian Mathieu à parler de l'espace comme 
une zone d'évaluation mutuelle ${ }^{33}$. Et ils entretiennent une distanciation, au moins discursive, avec l'espace partisan. Inspirée des travaux de Mathieu et des travaux de Laure Bereni sur l'espace des mobilisations pour la parité en politique, Delphine Lecombe offre une définition de «l'espace de la cause des droits des victimes » comme l'ensemble des collectifs - et leurs participants - qui luttent au nom des victimes et pour les victimes ${ }^{34}$. De la même veine, nous théorisons un « espace de la cause des proches de disparus » réunissant l'ensemble des collectifs et leurs participants qui luttent au nom des proches de disparus et pour eux. Cet espace, comme celui théorisé par Lecombe, «se caractérise par son hétérogénéité et sa transversalité : il est immergé dans d'autres espaces sociaux », tels que l'État, le mouvement bicommunautaire, les acteurs de sortie du conflit (organisations ou ONG internationales, bailleurs de fonds), le monde universitaire, etc. La description des différents acteurs en termes de pôles permet de prendre en compte le degré d' «immersion » d'autres univers sociaux dans cet espace et l'évolutivité des relations de coopération et de concurrence dans le temps et l'espace.

Nous retenons trois pôles: le pôle société civile autour de «l'Initiative bicommunautaire des proches de disparus, victimes des massacres et autres victimes des événements de 1963-1974 - Ensemble Nous Pouvons!» (ci-après décrit à l'aide de l'acronyme TWC, ou "Together We Can!"), le pôle « post-conflit» autour du Comité pour les personnes disparues (Committee on Missing Persons, $\mathrm{CMP}$ ) et un troisième pôle, le pôle «associations locales », qui inclue un réseau d'associations formées sur des dynamiques d'appartenance à un même lieu et qui renvoient à un événement de disparition particulier. Ces associations sont liées à l'Association des familles de martyrs ${ }^{35}$. Nous nous attachons particulièrement à rendre compte des dynamiques propres à ces trois pôles, la manière dont ils cherchent à politiser ou non la cause, et les modalités à travers lesquelles ils interagissent de manière à décrypter de manière fine les effets sociaux de la mise en place de ces initiatives.

33. Mathiev, 2007, p. 4.

34. LeCOMBE, 2014.

35. En 2009, quand la FEMED (Fédération méditerranéenne contre la disparition forcée) mène une visite exploratoire à Chypre, elle rencontre les deux comités historiques, le CMP, TWC, et deux associations de défense des droits de l'homme dirigés par des avocats, l'association gréco-chypriote Truth Now et l'Association turque chypriote des Droits de l'homme (KTIHD). Entretien avec la trésorière de la Femed, 22/05/16. 


\section{Construire une action dépolitisée : la renaissance du Comité pour les personnes disparues de Chypre}

From August 2004 until December 2005, the attitude of both sides vis-à-vis the Committee became more positive, with increased talks and agreed-upon confidence building measures to move forward with the exhumations, according to Wright. Since the end of 2005, plans to design and define the actual Project phases, establish who will be working in the scientific lab-including a team from the UK-based forensics group INFORCE, and determine equipment and personnel needs have been completed, forcing everyone's "nose to the ground," according to Wright. ${ }^{36}$

Cet extrait d'un télégramme diplomatique américain évoque la période de redémarrage du CMP, version 2004. Le projet suit le mandat de la première version, mais avec des moyens autrement plus importants. Des moyens financiers sont débloqués pour réaliser les objectifs officiels: la localisation, l'exhumation et l'identification des corps des disparus pour les rendre aux familles. Pour que l'institution et son action soient perçues comme apolitiques, elles sont toutes deux organisées de façon à ne pas mettre en cause le monopole des gouvernements sur l' « activité politique ». En s'inspirant des théories de Lagroye sur la dépolitisation ${ }^{37}$, on comprend que cela passe par un cloisonnement de la thématique de la disparition vis-à-vis des autres points d'achoppements intégrés dans les relations bilatérales, mais aussi vis-à-vis de la mémoire communautaire officielle. Cette dépolitisation passe par un système à trois branches, composé d'un principe de stricte réciprocité communautaire, du maintien d'un contrôle des autorités communautaires et d'un rôle technicien de contrôle des travaux exercé par des tiers « neutres ».

\section{Le principe de réciprocité}

L'ensemble des services et des actions du CMP sont marqués par un certain degré de réciprocité. Certains services qui avaient déjà été développés, comme les équipes de recherche, sont réorganisés de façon à respecter ce principe. Un département d'archéologie et un laboratoire d'anthropologie sont mis en place. Des investissements sont effectués pour utiliser la récente technologie d'identification ADN, utilisée trois ans plus tôt pour identifier les victimes du World Trade Center.

36. Public Library of US Diplomacy, 2007.

37. Lagroye, 2003. 
L'ensemble du personnel scientifique - 44 archéologues, 12 anthropologues et 2 généticiens - est embauché selon un principe de mixité communautaire: les équipes de travail sont composées à égalité de Turcs-Chypriotes et de GrecsChypriotes. La hiérarchie est également répartie en respectant ce principe: le responsable des équipes d'archéologie est turc-chypriote et celui du laboratoire est grec-chypriote. La réciprocité est maintenue dans le cadre de nouvelles activités ${ }^{38}$.

Même les choix relatifs aux sites de fouille et aux corps à examiner se font sur le principe de réciprocité, mais cette fois-ci, en veillant à ce qu'aucun des deux partis ne paraisse plus coupable d'exactions que l'autre. Ce principe se vérifie si l'on observe la constitution des listes et les proportions d'individus identifiés par le CMP. Dans ces deux cas, on peut observer un rapport plus ou moins constant d'un quart de Turcs-Chypriotes pour trois quarts de Grecs-Chypriotes, rapport qui correspond assez bien à la répartition communautaire d'avant-guerre ${ }^{39}$. Le maintien de cette proportion se fait de plusieurs manières. La liste turco-chypriote inclut notamment des individus victimes de massacres, mais dont la localisation des corps a toujours été connue ${ }^{40}$. En ce qui concerne les identifications, il semble que les exhumations ne suivent pas le rythme des identifications, ce qui pourrait être un moyen de maintenir l'équilibre des identifications en fonction de la communauté des victimes, maintenant ainsi un «équilibre de la barbarie » politiquement acceptable.

\section{Le maintien du rapport hiérarchique communautaire}

Au principe de réciprocité est associé un principe de maintien du rapport hiérarchique au sein des communautés. De façon générale, le personnel turcochypriote est sous la responsabilité et la direction du membre permanent turcochypriote et de ses assistants, de même pour les Grecs-Chypriotes ${ }^{41}$. Les membres eux-mêmes sont sous la tutelle directe de leur présidence respective. Les membres communautaires ont ainsi la responsabilité difficile de gérer l'équilibre entre

38. Ainsi, en 2007, trois psychologues grecs-chypriotes et deux psychologues turcschypriotes sont engagés dans le nouveau département de psychologie et en 2011, une seule photographe, turque-chypriote, sera engagée dans le laboratoire.

39. Les listes officielles du CMP reconnaissent 1510 disparus gréco-chypriotes et 492 disparus turco-chypriotes. Le 31 juillet 2018, le CMP a identifié 664 GrecsChypriotes et 225 Turcs-Chypriotes. Soit un rapport de 3/1 dans les deux cas.

40. Notamment les victimes de Muratağa-Sandallar-Atlilar.

41. Sur le terrain, si les choix sont pris en consensus, cela découle de cette volonté. C'est la souveraineté de l'État dans lequel se déroulent les fouilles qui prime et l'assistant du membre concerné décide en dernier lieu. 
74 Politique et sociétés à Chypre aujourd'hui

l'agenda politique officieux de leurs dirigeants politiques, de permettre le travail collectif au sein de l'institution et de maintenir les meilleures relations possibles avec les associations de proches de disparus et les proches directement.

Auteur: Donc diriez-vous que vous avez un poste autonome, ou que vous maintenez aussi des relations avec les autorités?

Membre permanent turco-chypriote: Bien sûr nous sommes autonomes, notre budget est autonome, notre travail est autonome, nos décisions sont autonomes, mais je peux dire, vu que nous sommes nommés par le politique... Bien sûr, ils promettent de ne pas faire de politique, ils n'interfèrent pas trop, mais aussi tu représentes ta société, tes familles. C'est une position très sensible tu as des obligations envers toutes les familles, parce que c'est pour elles que tu travailles, mais tu as des obligations aussi du fait des termes de références auxquels tu es attaché et tu as une liste, tu dois la respecter parce qu'elle est acceptée politiquement. Et tu es aussi responsable pour ton propre côté ${ }^{42}$.

\section{Le rôle pacificateur du tiers}

Ils sont aidés dans cette tâche d'équilibriste par les rôles tiers assurés par les organisations internationales. Ainsi, l'Union européenne prend en charge la grande majorité des quelque trois millions d'euros annuels de dépense moyenne du CMP. La formation des personnels, notamment des archéologues, a été assurée par une équipe argentine proche des milieux de promotion de la justice transitionnelle ${ }^{43}$. La gestion des conflits et de la cohésion de ces deux principes est maintenue par un tiers, le bureau du membre de l'ONU. C'est le secrétaire permanent qui, dans les faits, est chargé de transmettre aux employés les consignes données par un supérieur de l'autre communauté.

Auteur: Vous n'êtes pas un membre?

Secrétaire permanent du CMP: Non, je suis aussi le secrétaire permanent du CMP je veille à ce que les décisions prises soient suivies, mises en place. C'est en gros ma fonction principale. Le problème c'est que l'institution est bicommunautaire, mais quand

42. PlÜMER KüÇÜK, 2016.

43. Les équipes d'archéologie sont formées par l'Equipo Argentino de Antropología Forense (EAAF), une équipe d'archéologues, indépendante spécialisée dans la recherche des disparus. Pour la formation des équipes de fouilles de fosses de «disparus» indépendantes en contexte espagnol, voir SMAOUi,2015. 
on en vient aux chaines de commandement les Grecs-Chypriotes vont reporter aux autorités grecques-chypriotes, les TurcsChypriotes aux autorités turques-chypriotes, donc c'est très difficile pour un Grec-Chypriote de donner des ordres à un Turc-Chypriote. C'est là que se situe mon rôle, je n'appartiens à aucun côté, je peux donner des instructions à tous sans qu'il ne le questionne. Donc... C'est les membres qui décident, mais pour la mise en place, quand c'est sensible, c'est plus pratique que ça soit moi, plutôt que quelqu'un d'une des communautés, qui le fasse ${ }^{44}$.

Dans certaines occasions, l'apparition de déséquilibres entraîne la neutralisation d'activités: par exemple, lorsque le laboratoire de test génétique est mis en place, il est le seul laboratoire compétent sur l'ile, et il est en République de Chypre. Ce déséquilibre entraîne une série de conflits internes qui aboutissent, par consensus, à la délocalisation des activités à l'étranger ${ }^{45}$. Ces mécanismes de dépolitisation semblent avoir eu un succès certain, puisque l'entreprise de localisation, d'identification et de réinhumation des corps connaît un succès indéniable, avec plus de la moitié des personnes sur la liste qui ont été exhumées entre 2006 et 2018 et plus d'un tiers qui ont été identifiés et dont la réinhumation a été effectuée ou est programmée.

De même, le CMP semble avoir réussi avec succès à créer un espace de rencontre «apolitique » des dirigeants communautaires, utile en temps de crise. Entre la première rencontre bilatérale de haut niveau post-référendum en 2006 et 2018, la visite du CMP a réuni à de nombreuses occasions les deux présidents. La visite du laboratoire du CMP est devenue un des passages obligatoires de toutes les visites de représentants de l'ONU ou de délégations internationales.

Par ailleurs, l'organisation du CMP crée une situation inédite de collaboration bicommunautaire quotidienne à travers le travail. La mise en place d'équipes mixtes, une première pour des employés publics sur l'île, crée un précédent, et les employés, transformés par «l'expérience socialisante de la fosse ${ }^{46} »$, formulent des retours positifs sur cette collaboration qui contribuent à rendre crédible une vie en commun. Le mode de fonctionnement n'est pas non plus imperméable à des ponts entre l'engagement professionnel «dépolitisé » et l'engagement militant. Certains de ces employés manifestent par exemple un rapport d'allégeance

44. Von KöNIG, 2016.

45. Dans une première période par le laboratoire de l'International Center for Missing Persons géré par l'ONU en Bosnie puis, aujourd'hui, par un laboratoire privé américain. 46. Smaoui, 2015. 
qui dépasse l'échelle communautaire, en se considérant comme employé de l'organisation internationale CMP et formulent dans le cadre de leur travail des idées contraires aux principes du CMP, notamment autour de la question de la justice. Ce cadrage «apolitique» entraîne toutefois des relations complexes avec les autres pôles de la cause des disparus, notamment le pôle constitué d'une association engagée au sein du mouvement bicommunautaire Together We Can!

\title{
Together We Can! : un mouvement né d'une rencontre entre «société civile » et parcours individuels de proches de disparus
}

Together We Can! est officiellement enregistré en 2010 par treize proches de disparus, dont six Turcs-Chypriotes et quatre Grecs-Chypriotes ${ }^{47}$, mais a commencé à militer en dehors d'une structure officielle à partir de 2006. C'est la première association généraliste ${ }^{48}$ de défense des intérêts des proches de disparus qui accueille des membres des deux communautés. C'est une particularité revendiquée, au centre de son inscription militante et qui s'associe avec une volonté de se distinguer des deux associations progouvernementales communautaires existantes jusque-là, le Comité panchypriote des prisonniers disparus (gréco-chypriote) et l'Association des familles de martyrs (turcochypriote), comme l'explique Hüseyin Akansoy, l'un des deux fondateurs:

Des deux côtés, il y a deux associations de familles. Elles ont des buts précis. Le premier but est de renforcer les différences entre

\begin{abstract}
47. Il est difficile d'évaluer le nombre exact de membres actuels et ses fluctuations, pour deux raisons. Premièrement, les données sur l'adhésion ne sont pas communiquées parce que l'association ne fonctionne pas sur un modèle économique fondé sur la cotisation. L'adhésion des treize personnes en 2010, aux dires des responsables interrogés, ne correspond qu'à une obligation légale de remplir des postes administratifs. L'association fonctionne au sein d'un réseau large de sympathisants et de militants inscrits dans d'autres causes. Par ailleurs, les postes officiels prennent une importance secondaire en pratique. La deuxième raison, qui n'est pas donnée ouvertement, correspond à l'incapacité de mobiliser au-delà d'un cercle militant proche et à la volonté de représenter l'ensemble des proches de victimes, qui ne sont pas engagés de manière générale. Parmi les militants présentés comme membres de l'initiative dans les conférences auxquelles nous avons assisté ou dont nous avons eu connaissance, certains n'étaient pas sur la liste de 2010. D'autres sur la liste n'ont pas semblé être actifs aujourd'hui : ils n'étaient pas mentionnés par les interrogés et n'ont pas non plus été présentés pendant les conférences. Enfin, certaines personnes actives avant l'enregistrement officiel ne sont pas non plus sur la liste. Les membres actifs après 2010 ne sont donc probablement pas membres officiels. Ce nombre de militants semble néanmoins constant sur la décennie d'activité de l'association.
\end{abstract}

48. Nous parlons d'associations généralistes quand elles ne sont pas spécifiquement organisées autour d'une localité ou d'un massacre/kidnapping particulier. 
les deux côtés. Ils ne se sont occupés que de leur communauté et diffusent une propagande uniquement fondée sur ce que l'autre aurait fait. Pendant des années, ils ont diffusé ce poison. [...] Ceux qui soutenaient la paix, ceux qui étaient au-devant des demandes de réunification du côté turc, qui s'intéressaient aux questions des martyrs, des disparus, se sont retrouvés. Ils étaient à la recherche d'une nouvelle voix. S'il y avait eu une autre organisation, nous n'en serions pas là ${ }^{49}$ !

TWC se définit comme un comité de victimes, un mouvement "grassroot", c'est-à-dire composé d'individus directement concernés, et pensé dans une dynamique par le bas. Elle propose de créer des occasions pour les proches de victimes de partager leurs expériences, de recueillir des informations sur la localisation des fosses communes, d'organiser des évènements pour sensibiliser l'opinion publique aux responsabilités partagées des crimes de guerre à Chypre, et d'intervenir auprès des jeunes générations.

La fin des barrages matériels à la rencontre et aux échanges, notamment l'ouverture des frontières a permis l'aboutissement du processus de collectivisation des victimes, matérialisé par la création de TWC et l'apparition d'un discours de promotion du « disparu chypriote » qui remplace les étiquettes communautaires. Ce discours s'appuie sur une politisation de la question. La définition du « disparu chypriote » ou de la «victime chypriote » s'appuie sur la «définition d'une expérience partagée et communicable de la disparition d'un proche, à laquelle sont associées des intentions, des conséquences et des responsables ${ }^{50}$.» Aujourd'hui, TWC est la seule organisation à défendre activement les droits des proches de disparus dans leur ensemble, en tant que comité de victimes. Pourtant, paradoxalement, le nombre de personnes activement engagées dans ses activités est resté constamment faible, et l'association n'a jamais eu vocation à faire adhérer un grand nombre de personnes. À la différence des autres comités, TWC a choisi de ne pas accueillir de membres sympathisants, dont l'adhésion serait essentiellement symbolique, et limite l'accès aux militants actifs. La population membre est d'ailleurs composée en majorité de membres «historiques», engagés dans le projet avant la fondation officielle. Une attention particulière à ses membres, sous la forme d'une analyse de trajectoires, permet de mieux comprendre les objectifs militants du groupe et son inscription dans le paysage politique.

49. Akansoy, 2016.

50. Diaz \& Gutiérrez Ruiz, 2009. 
78 Politique et sociétés à Chypre aujourd'hui

Le groupe fondateur de TWC se constitue entre 2001 et 2006 autour de deux réseaux d'activistes. Les enquêtes de Sevgül Uludag et les visites régulières qu'elle organise pour les proches de disparus et de victimes de massacre favorisent le développement d'un premier réseau. En 2005, elle publie son livre Oysters With the Missing Pearls, untold stories about missing persons, mass graves and memories from the past, qui réunit les articles qu'elle a écrits sur les victimes et leurs proches. Ce best-seller à l'échelle de l'île, traduit en anglais en 2006 et en rupture de stock aujourd'hui, l'a rendu célèbre comme «la » journaliste qui traite de la question des disparus, bien que ses articles ne parlent pas toujours de disparus, deux tiers d'entre eux portent sur des assassinats sans disparition, des réflexions sur la violence et des textes mettant en valeur le passé idéalisé d'avant-guerre. Ce cadre persiste aujourd'hui et si la disparition représente le thème le plus visible de son travail et son fil conducteur, il n'englobe qu'une partie de son œuvre.

La particularité du réseau développé par Uludag est de réunir des gens qui ne se connaissent pas à la base. Ce réseau en étoile se complexifie par la suite, lorsque les proches se rencontrent au cours de la présentation de la version anglaise de son livre. À chaque présentation, elle se fait accompagner de proches de disparus et d'autres gens qu'elle a interviewés, pour qu'ils parlent de leur expérience au public.

À chaque fois que je suis allée parler du livre, j'avais toujours deux proches de disparus, un Grec-Chypriote et un Turc-Chypriote, pour montrer qu'ils sont amis et qu'ils cherchaient des informations sur les lieux d'inhumation ${ }^{51}$.

Ce mode de fonctionnement, en apparence anodin, permet la création de relations entre proches de disparus tout en leur offrant une première expérience de la scène et l'occasion de développer les premières routines d'un répertoire d'action militant. En effet, ceux qui accompagnent les lectures n'ont pas de carrière militante collective derrière eux.

De manière parallèle, un deuxième réseau se construit autour de deux militants du mouvement bicommunautaire. À partir de 2004, d'autres proches de disparus ou de victimes de massacres organisent des rencontres informelles, rendez-vous organisés à l'initiative de Christos Eftymiou et Hüseyin Akansoy pour réfléchir aux actions qui pourraient être entreprises autour de la question résurgente des disparus dans le prisme de l'action bicommunautaire. Entre dix

51. UludaĞ, 2016. 
et quinze proches de disparus et de victimes du conflit ${ }^{52}$ qui seraient prêts à se mobiliser autour de cette identité victimaire pour porter une action collective se réunissent régulièrement entre 2004 et 2006. Ils se sont rencontrés à travers des réseaux militants propres au mouvement bicommunautaire. La démarche est claire : c'est la réflexion autour de la poursuite et de la réinvention du mouvement bicommunautaire qui nourrit la réflexion autour de la disparition.

La rencontre de ces deux réseaux favorise une redéfinition substantielle de la disparition, à travers la nécessité de trouver un langage à la fois conforme aux objectifs communs et susceptible de traduire les spécificités de chaque cas, et les agendas politiques de chaque membre ${ }^{53}$. La constitution d'une communauté de destin ${ }^{54}$ autour d'une intention de montée en généralité a abouti à la promotion d'une nouvelle catégorie, les «proches de disparus, victimes de massacres et autres victimes des évènements de 1963-1974». Cette genèse a produit également une autre des particularités de TWC, qui est de s'inscrire dans le mouvement bicommunautaire. Comme les autres associations généralistes, TWC parle au nom de tous les proches de disparus et, plus largement, au nom de l'ensemble des individus qui ont souffert de la «Question chypriote ». Mais elle le fait en participant à des événements réguliers auprès d'autres associations bicommunautaires et auprès de partis politiques et de syndicats soutenant la cause, en signant des mémorandums communs et, on l'a vu, une partie de ses membres actifs a milité ou milite dans d'autres associations bicommunautaires. Cette identité bicommunautaire est également visible dans les objectifs affichés de l'association, notamment la revendication d'un rôle de sensibilisation aux responsabilités partagées des crimes de guerre sur l'île et un répertoire militant qui fait une part belle aux interventions dans les écoles dans le but de toucher les nouvelles générations.

TWC se distingue du reste du mouvement, néanmoins, par le rôle plus ou moins actif de certains profils qui ne présentent pas de socialisation militante préalable. Un petit nombre de ses proches participent aux évènements publics en faisant le récit de leurs pertes et en appelant à la reconnaissance des souffrances des habitants de Chypre. D'autres participent de manière plus anecdotique, en

52. Cette estimation est fondée sur les entretiens avec les membres de l'association, sur les statuts officiels de l'association et sur l'exploitation d'archives journalistiques entre 2004 et 2007 .

53. En s'inspirant, sans se revendiquer clairement, du vocabulaire développé par les auteurs promouvant la justice transitionnelle comme solution à la question de la disparition.

54. Diaz \& GutiérRez Ruiz, 2009. 
répondant à l'appel formulé par Sevgül Uludag de transmettre des informations susceptibles de pouvoir identifier les lieux où sont enterrés les disparus. Ces profils permettent à l'association de se revendiquer comme une association grassroot, même si l'engagement des cadres dirigeants tire sa source du militantisme bicommunautaire en premier lieu, parfois dans le cadre d'un emploi rémunéré, et même si ces derniers militent dans des réseaux partisans. Néanmoins, l'analyse des trajectoires individuelles des membres actifs de TWC permet de tracer des parallèles entre les militants issus du mouvement bicommunautaire et les proches de disparus. On retrouve chez la plupart des militants l'expérience de la perte d'un membre de la famille à un jeune âge et la convocation d'une éducation positive vis-à-vis de l'autre communauté et de relations familiales intercommunautaires.

L'existence de ces deux pôles, le pôle « société civile » et le pôle « post-conflit » a été l'occasion de coopération, mais également de tensions. La dépolitisation de la thématique de la disparition, qui a permis la relance du CMP, a de fait créé une opportunité de réengagement pour un ensemble de militants après l'échec du référendum de 2004. Dans cette configuration, l'entrée des proches de disparus turco-chypriotes, militants néophytes, répond à des logiques indépendantes de leur socialisation et fait figure d'exception. Le cadrage spécifique qui a permis d'embarquer la cause des disparus dans un ensemble plus grand a conduit à une forme de re-politisation de la question: au lieu d'être traité comme une cause humanitaire appartenant à un passé révolu, le travail de TWC donne à montrer une continuité idéologique et historique entre les responsables du conflit d'hier et les responsables de la division d'aujourd'hui. C'est-à-dire que l'engagement dans cette cause sert non seulement à désigner les responsables des disparitions, mais aussi les responsables politiques des blocages quant au traitement de la question (les autorités), des acteurs envers lesquels beaucoup de militants de la cause des disparus ont eu une expérience conflictuelle par le passé, une vision qui entre en conflit avec la volonté du CMP de ne pas identifier de coupable et de s'en tenir à identifier les corps des disparus.

La confrontation des deux pôles entraîne également des tensions sur le terrain. La mise en place par Sevgül Uludag d'un service parallèle de récolte d'informations sur la localisation des corps reçoit au début l'appui informel du membre turco-chypriote intérimaire du CMP, Ahmet Erdengiz, entre 2004 et 2006. Mais cette activité de récolte d'informations associée à des déplacements non concertés sur le terrain de Sevgül Uludag produisent à plusieurs reprises de fortes tensions, notamment lorsque les habitants s'opposent à l'enquête de la journaliste. L'intensification des tensions conduit à un recadrage et le nouveau membre turco-chypriote, Gülden Plümer Küçük, nommé en 2007, adopte une position plus distanciée, reconnaissant la qualité du travail de la journaliste, tout 
en soulignant les risques que cette activité fait peser sur le maintien de l'activité « humanitaire » et apaisée du CMP ${ }^{55}$.

La mise en place de la dépolitisation du CMP passe ainsi par une mise en concurrence, au sein de l'espace de la cause des disparus, entre un pôle «société civile » en voie de reconfiguration après avoir atteint des limites dans sa stratégie primaire, et un pôle «post-conflit» en quête d'affirmation ${ }^{56}$. La routinisation des activités du CMP pendant les années suivantes témoigne de l'imposition d'un registre propre à la dépolitisation, notamment l'utilisation d'un vocabulaire spécifique créant des passerelles émotionnelles avec les familles: on parle par exemple peu de disparus, mais plutôt de "missing" (personnes manquantes) ou de "loved ones" (êtres aimés). Mais le recours au pouvoir politique dans certains cas entraîne également un phénomène de rejet de la part du pôle «société civile ». Un phénomène de distanciation d'acteurs, qui se présentent comme acteurs de terrain, avec une certaine perception du politique, a notamment été étudié par Johanna Siméant dans son travail sur le rapport au politique des travailleurs expatriés de l'humanitaire ${ }^{57}$.

La stratégie de la dépolitisation permet un fonctionnement relativement efficace du CMP, qui conduit depuis 10 ans des fouilles et des identifications régulières. La stratégie atteint pourtant des limites qui ont pu circonscrire sa capacité d'action. L'organisation a par exemple renoncé à certains terrains de fouille face aux pressions des locaux pour éviter d'afficher les tensions. Le rapport institutionnel qu'elle entretient avec les familles semble aussi donner selon l'époque moins de résultats que l'approche de Sevgül Uludağ pour obtenir des informations sur la localisation des corps. Ce relatif échec de l'entreprise de séduction du public peut découler d'une certaine appréhension face à la machine institutionnelle de la part d'individus coupables, dans une certaine mesure, d'avoir gardé une telle information pendant plusieurs décennies, appréhension que la mise en place d'une relation de confiance à l'épreuve de plusieurs années permet de dépasser.

55. Public Library of US Diplomacy, 2007.

56. La notion de mise en concurrence renvoie à la notion de cycle proposée par Tarrow pour décrire les phénomènes de croissance et décroissance des activités collectives. Elle n'est pas convoquée ici directement mais a servi d'inspiration. Voir Isabelle Sommier, in Fillieule \& Mathieu, 2009.

57. SimÉANT, 2003. 
82 Politique et sociétés à Chypre aujourd'hui

\section{Investissement des proches de disparus}

Mais quid, dans tout ça, des proches de disparus ? Nous avons vu dans la première partie qu'ils n'avaient pas réalisé de mouvements collectifs de revendications dans la période pré-référendum. Aujourd'hui, à l'exception des quelques proches impliqués dans les activités de TWC, il semble que la plupart ne soient toujours pas engagés dans des activités militantes. Il faut noter toutefois certaines organisations, qui ont pris la forme de résistance, selon l'époque et les individus concernés, face aux activités du CMP et actuellement, d'un investissement dans la monumentalisation de la mémoire de la disparition et des massacres. Cette patrimonialisation segmentée montre l'absence d'une montée en généralité autour de la disparition et d'un maintien d'identités localisées.

Comme nous l'avons vu, des deux côtés de l'île, les proches de disparus se voient privés de l'accès à des informations essentielles sur le devenir de leurs proches. À la fin des années 1990, la découverte de 100 corps de disparus grécochypriotes dans un cimetière contrôlé par la République de Chypre provoque un scandale retentissant qui entraîne pour la première fois la reconnaissance officielle par les autorités gréco-chypriotes du décès, d'une partie au moins, des Agnooumeni, officiellement toujours prisonniers de guerre en Turquie. En « République turque de Chypre du Nord », certains proches de disparus turcochypriotes, minoritaires, se lancent dans des recherches individuelles qui sont rendues difficiles par les autorités. Le bureau du CMP, avant 1997, est en zone tampon sous contrôle de l'ONU, accessible grâce à un permis donné aléatoirement par les autorités turco-chypriotes et aucun effort n'est fait pour faire connaître son existence. Dans les rares cas où des proches de disparus ont réussi à entrer en contact avec les représentants turco-chypriotes de l'époque, ces derniers ont nié jusqu'à l'existence de fosses. Dans le cas où les proches parviennent à localiser les lieux potentiels des fosses, l'accès leur est refusé. Par ailleurs, au cours des années 1990, des déplacements de fosses couverts par les autorités ont eu lieu ${ }^{58}$. Avant 2004, il semble impossible pour les démarches individuelles d'obtenir le soutien des autorités turco-chypriotes.

Après 2004, la situation se retourne: les autorités sont demandeuses de la coopération des proches de disparus, dans un premier temps pour obtenir des prélèvements $\mathrm{ADN}$ et, dans un deuxième temps, pour participer au processus de reconnaissance des corps et de réinhumation.

58. Témoignage de Sevilay Berk rédigé par la journaliste Sevgül Uludag https://www. stwing.upenn.edu/ durduran/hamambocu/authors/svg/svg7_3_2004.html 
Les premières campagnes $\mathrm{ADN}$ ont un résultat mitigé. Certains proches acceptent très rapidement, alors que d'autres refusent dans un premier temps. La réponse négative la plus médiatisée à l'époque, celle des proches des disparus d'Aleminyo fait apparaître les lignes de fractures majeures des disparus. Formulée par Küdret Ö, fils de disparu et à l'époque membre des équipes de négociations, elle montre le poids que donne la capacité à agir en collectif, poids dont ne disposent pas les proches de disparus individualisés. Deuxièmement, le refus est motivé par des justifications politiques, notamment la localisation initiale du laboratoire de test ADN en République de Chypre. Pour finir, le refus souligne la différence entre les missing « officiels » et les missing « disparus ». La construction des listes a favorisé l'inclusion dans les listes d'individus dont la localisation des corps a toujours été connue. Le processus d'identification et de réinhumation formel peut ainsi être perçu comme découlant moins d'une démarche humanitaire que d'un réinvestissement politique des conséquences de la guerre et d'une volonté de maintenir un «équilibre de la barbarie » artificiel. Par ailleurs, on remarque que certains des proches de missing «disparus» ont entamé des démarches personnelles dès l'ouverture du mur, en allant à la rencontre du comité grécochypriote qui était resté ouvert, et en retournant dans leur village d'origine pour d'ultimes vérifications, comme les proches des disparus du village de Vouno.

Courant 2005, les procédures de récolte des prélèvements ADN des proches de disparus, auparavant gérées par un laboratoire gréco-chypriote, sont récupérées par le CMP et un laboratoire turco-chypriote est commissionné pour œuvrer aux prélèvements pour les proches du Nord. Ce changement permet d'acquérir le soutien des proches qui avaient auparavant refusé et contribue ainsi à dépolitiser les pratiques de collecte de l'ADN. La remobilisation des proches se fait également à travers le "viewing" et la réinhumation. Le "viewing" est le nom donné par les employés du CMP à la « cérémonie » de présentation des corps, les proches sont invités au laboratoire du CMP où un membre de leur communauté de chaque équipe leur présente le processus qui a amené à retrouver le corps. Ils sont ensuite confrontés, s'ils le souhaitent, aux restes de leurs proches. L'expérience de la confrontation se vit alors dans une relation individuelle ou familiale qui peut être traumatisante pour certains mais qui participe à donner une reconnaissance officielle aux proches.

Les cérémonies de réinhumation constituent elles une remise à l'agenda sur la place publique des événements de 1963-1974 et sont gérées au sein de la communauté. Elles procèdent à une réécriture de la mémoire qui place le proche de disparus au centre du dispositif. La perspective de la cérémonie le place dans une situation nouvelle de décisionnaire. Il peut choisir d'opter pour une cérémonie militaire ou une cérémonie privée; dans le cas où il choisirait la cérémonie militaire, 
il se retrouve au centre d'une cérémonie nationale qui impose une montée en généralité et qui devient l'occasion de tentatives de politisation de la part des autorités. Ces cérémonies se déroulent pour la plupart dans le cadre militaire. Elles s'inspirent des rituels militaires turcs républicains: phénomène républicain par excellence, elles témoignent du respect de la nation et de l'entrée du décédé dans la communauté des martyrs. L'attribution du statut de martyr aux proches de disparus en 2002 prend alors tout son sens. En l'absence de corps, les honneurs de la nation restent incomplets. Elles sont l'occasion pour l'ancienne « coalition régnante » issue des mücahit, écartée du pouvoir, de se repositionner sur la place publique. Ces cérémonies prévoient la tenue d'un discours dont la maîtrise est l'objet d'enjeux de re-politisation. L'Association des familles de martyrs y occupe une place régulière. Le président Ertan Ersan ${ }^{59}$ donne un discours qui reprend les termes régulièrement convoqués pour décrire le conflit: « Rums ${ }^{60}$ Barbares », « génocide», « nous ne pouvons pas vivre avec eux ». Elle n'est pourtant pas une obligation. Plusieurs proches de disparus qui font le choix d'une cérémonie militaire s'y refusent et prennent la parole dans une logique de défiance :

Auteur: Il y a un comité des familles de martyrs.

Sevilay: Mon petit, ce mec, il est malhonnête, c'est un voleur. Il utilise les martyrs pour de l'argent. Hé! Écoute, on a trouvé la dépouille de mes parents hein, on a fait une cérémonie officielle, militaire, pour les enterrer. Cet homme [Ersan] voulait faire un discours, moi devant tous les soldats, j'ai demandé qu'il n'emmène même pas ses potes, parce qu'il veut parler pour montrer qu'il a travaillé pour trouver les os. Il veut se faire de l'argent de ma douleur. Il veut dire « Moi je les ai trouvés! » ce genre de mensonge. J'ai dit que je ne veux pas de cette personne. J'ai parlé, pas lui. J'ai fait comme ça (rires) ${ }^{61}$.

La perspective d'avoir un proche enterré comme « martyr » constitue pour d'autres une motivation importante. Ainsi, Zilliha Uluboy relève un cas limite qui intéresse notre développement. Quatre corps de disparus n'avaient pas été signalés par leurs proches comme martyrs au sortir de la loi en 2002 avant la

59. Ertan Ersan a été remplacé courant 2018 à la tête de l'association après son décès.

60. Le mot «Rum», qui désigne les populations grecques orthodoxes de l'Empire ottoman, est utilisé dans la communauté turco-chypriote pour désigner les GrecsChypriotes. Pour désigner les Grecs de Grèce, ils utilisent le mot turc «Yunanlı», comme en Turquie.

61. B. 2016. 
date limite imposée par la loi. Lorsqu'ils ont été identifiés, n'étant pas dans la liste officielle, ils ne pouvaient bénéficier d'un enterrement militaire. La famille a souhaité attendre le changement de la loi avant de récupérer les corps, de manière à assurer un enterrement militaire officiel à ses proches ${ }^{62}$.

L'enterrement est également l'occasion d'une mise en spécification souhaitée. Le choix du lieu d'enterrement est dans certains cas l'occasion d'une mobilisation de ressources en vue de pérenniser l'histoire particulière qu'ont traversé les disparus, mobilisation qui souligne encore une fois une très forte disparité entre les disparus «individualisés » et les disparitions de groupe. Si certains disparus, notamment ceux qui ont fait l'objet d'enlèvements «individualisés », sont enterrés au cimetière des martyrs-disparus de Nicosie ou dans des carrés de martyrs périphériques, depuis 2008, quatre mausolées propres aux cas de disparitions massives ont fait leur apparition dans le paysage turco-chypriote. Ces lieux de mémoire sont associés à un musée qui expose les objets personnels retrouvés avec les disparus et vise à entretenir leur mémoire.

Après la première cérémonie, les réticences des proches de disparus s'émoussent. Le succès de la cérémonie semble avoir emporté les adhésions et rassuré quant au traitement des corps par le CMP. Les proches de disparus interrogés sont unanimes pour déclarer avoir ressenti un soulagement après la réinhumation de leurs proches, y compris des proches de missing «officiels». Les proches de missing «disparus » expliquent leur soulagement par la certitude trouvée de la mort de leur proche et la fin d'une attente qui n'avait jamais cessé.

Ce soulagement entre en résonnance avec la littérature portant sur la justice transitionnelle qui influence à la fois le travail du CMP et de TWC et plus largement l'ensemble des processus d'identification de disparus qui sont conduits à travers le monde dans le cadre de procédure de post-conflit ou de réconciliation nationale depuis les années $1990^{63}$. On trouve notamment un discours très influencé par les concepts de résilience et par la relation entre une blessure individuelle ouverte et l'incapacité d'une société à se soigner. Un des totems des travaux sur la justice transitionnelle est la capacité de formes de para-justice qui mettent les victimes au centre du processus, à apporter une forme de soulagement et la capacité à tourner la page. Le concept de «deuil différé », convoqué par les psychologues qui ont travaillé sur la disparition et les professionnels de la justice transitionnelle entre ici en résonnance avec le témoignage des proches.

62. Uluboy, 2016.

63. LeFranc \& Mathieu 2009. 
Auteur: Les gens disent que ça permet de tourner la page. Vous en pensez quoi ?

A.G. : Mon père est mort, je peux le visiter sur la tombe, donner des fleurs, lire la prière des morts, pour nous c'est une bonne chose: mon père est là. Tu sais, il est là, il est mort. Mais si tu ne sais pas, tu as toujours une attente, il doit être mort puisqu' il ne vient pas, mais tu ne peux pas être sûr ${ }^{64}$.

Sevilay: Maintenant je suis heureuse. Il y a une tombe, j'ai mis des fleurs, on fait la prière, c'est très beau, je suis heureuse. Pouvoir avoir un lieu de visite, faire les rituels constitue une source de soulagement pour les disparus ${ }^{65}$.

Auteur: Retrouver ses restes était-il quelque chose d'important pour vous?

O. P.: C'était important. Premièrement, il y a une tombe maintenant. Nous savons où il est maintenant. Avant nous ne savions pas. Maintenant, nous savons où il est, nous avons pu prier pour lui et l'enterrer. Nous pouvons aller le voir pour les fêtes et pour le saluer ${ }^{66}$.

Ce soulagement est aussi le résultat d'une quête aboutie de normalisation. L'exemple des cercueils souligne cette soif de normalisation. Le fait que les cercueils utilisés par le CMP soient plus petits que les cercueils traditionnels, parce qu'ils sont construits pour stocker des os et non des corps, est une des critiques les plus régulières que les proches formulent, car ces cercueils différencient visuellement l'être cher enterré des défunts «normaux ».

\section{La création d'associations de défense du souvenir des martyrs: une collectivisation relative de la cause}

La généralisation de ces procédures et leur promotion par les autorités communautaires permet le lancement d'un processus d'investissement par les proches de la thématique de la disparition. À l'exception des quelques proches investis dans TWC ou dans d'autres activités bicommunautaires, cet investissement prend moins la forme de revendications judiciaires que d'une démarche de monumentalisation de la disparition. L'entretien de ces cimetières

64. G, 2016.

65. B, 2016.

66. A, E, I Palabiyik, 2016. 
et le travail de mémoire sont intimement associés dans ces projets. Ils constituent un moteur pour la mise en place d'associations à partir de la fin des années 2000. À partir de 2007, des associations représentatives de ces groupes sont créées, dans le cadre de l'Association des familles de martyrs. Aujourd'hui, quatre associations principales existent au sein de la communauté turque chypriote: l'Association des martyrs de Dochni, l'Association des martyrs d'Aleminyo, l'Association des martyrs de l'autobus perdu et l'Association des martyrs de Muratağa-SandallarAtlılar. La mémorialisation et la reconnaissance est leur objectif principal. Elles s'investissent dans la construction de şehitlik (cimetières de martyrs) spécifiques pour chaque groupe de disparus. Dans cette optique, les travaux d'identifications du CMP sont perçus comme une occasion de revaloriser la mémoire des victimes, même dans les cas où elles ne constituaient pas des disparus à proprement parler comme à Muratağa-Sandallar-Atlılar, les dirigeants considérant que le besoin d'identification relève d'une reconnaissance internationale qui «est bonne pour la reconnaissance du génocide ${ }^{67} \gg$. La constitution de ces organisations a également permis un retour «dans le jeu» de l'Association des familles de martyrs, sous l'égide de laquelle ces organisations sont fondées. Elle organise des réunions régulières avec les dirigeants de ces associations, les conseille et leur fait bénéficier ses réseaux.

Un autre fait important que ces mécanismes donnent à voir est la différenciation produite par l'expérience collective de la disparition. L'ensemble des mausolées spécifiques qui ont été construits concerne des cas où les disparus partageaient des liens de parenté et le même village, des liens pour rapprocher les associations qui portent ces mausolées de la logique qui sous-tend les associations de hemşehri, des associations visant à promouvoir et à maintenir les ressources du village en consolidant les liens d'origine localement ou dans les lieux de diaspora des villageois. Les solidarités qui se jouent ici sont ancrées dans l'expérience traumatique de la disparition, mais reflètent également ces logiques de valorisation de l'identité locale. Ces phénomènes montrent une différenciation des engagements et des perspectives de montée en généralité des disparus. Si les proches de « massacrés » se re-polarisent sur leur espace « communautaire » pour entretenir la mémoire particulière de leurs morts, les proches de disparus enlevés en 1963 n'ont pas su se collectiviser pour prétendre à un tel objectif. Les différences ne tiennent pas seulement à la distinction entre victimes individuelles et victimes collectives, qui peuvent s'appuyer sur des relais. Elles reposent sur plusieurs paradigmes: l'engagement politique, la perception de la place de la Turquie, le désir d'être un «proche de martyr», la cible des rancœurs entretenues... On

67. A et G, 2016. 
conclura que la certitude obtenue de la mort, à la suite des exhumations successives, a donné une certaine légitimité aux proches de disparus en leur rendant un corps non seulement physique, mais social.

\section{Conclusion}

Le présent article nous a permis de rendre compte, à travers une analyse sociohistorique dans le temps long, des dynamiques qui sous-tendent l'apparition d'une cause victimaire autour de la disparition au sein de la communauté turcochypriote, près de trente ans après les dernières disparitions. La deuxième partie a permis d'entrer dans le détail et de s'intéresser à l'impact croisé des logiques militantes et politiques des différents pôles investis dans la cause et des trajectoires individuelles des personnes qui agissent au sein de ces pôles. Elle montre comment des objectifs politiques et sociaux différenciés entraînent une tension autour de la définition de la cause et des conséquences politiques et judiciaires qui doivent en être tirées. On peut résumer cette opposition entre les tenants d'une « cause des disparus » qui militent pour une finalité judiciaire qui viserait les responsables des crimes, et les soutiens d'une «cause des proches de disparus » pour qui l'objectif des enquêtes est d'abord d'apporter du soulagement aux victimes. Au sein de ces derniers, on peut distinguer deux groupes. Les acteurs politiques qui soutiennent le CMP et qui y voient un moyen de maintenir un canal de discussion entre les partenaires des négociations pour la réunification de l'île et les proches de disparus engagés dans des associations locales qui y voient un moyen d'investir leur traumatisme à travers un travail de mémoire et de monumentalisation/ patrimonialisation.

Les phénomènes de dépolitisation-repolitisation qui constituent un des fils rouges de cet article soulignent la difficulté d'appréhender de tels processus. La contribution, liminaire, de cet article à la compréhension de ces phénomènes se concentre sur les effets secondaires de la mise en place de la dépolitisation dans le cadre des politiques de post-conflit. Dans le cas que nous avons étudié, la mise en place du Comité pour les personnes disparues découle d'une volonté des partenaires internationaux, et de l'ONU en particulier, de trouver une parade à l'échec du processus de réunification en adoptant les méthodes de post-conflit nées des expériences de justice transitionnelle. Les partenaires internationaux concluent alors que le référendum de 2004 a échoué parce que les termes de son plan ont été perçues comme dépassant des lignes rouges politiques. L'identification des disparus pour un but affiché comme «humanitaire» est donc conçue pour ne pas s'inscrire en porte-à-faux de ces lignes rouges. Le résultat, 14 ans après l'annonce de la relance et 12 ans après les premières réinhumations, est 
mitigé. Si le CMP représente toujours aujourd'hui le seul exemple d'entreprise bicommunautaire soutenue par les deux gouvernements de l'île et peut se targuer d'avoir identifié un très grand nombre de disparus, l'entreprise de dépolitisation officielle n'a pas éliminé l'utilisation politique de la disparition par les dirigeants communautaires. Au contraire, elle a permis un réinvestissement de la mémoire de la guerre et un renforcement de la coalition régnante issue de la guerre, en réinsérant dans le quotidien un rituel d'inhumation militaire qui redonne de la vie à un culte des martyrs qui n'était plus nourri depuis 1974. En parallèle, l'humanitarisation de la cause de la disparition n'a pas empêché des individus et des groupes pro-réunification de se saisir de cette cause pour dénoncer les crimes commis et couverts au nom du nationalisme à Chypre. Ces acteurs ont réussi, à travers la journaliste militante Sevgül Uludag et dans une moindre mesure à travers le groupe de proches de victimes bicommunautaires Together We Can!, à s'appuyer sur la visibilité du CMP pour obtenir une vitrine internationale à travers laquelle ils appellent à juger les responsables des crimes et leurs complices, tout en soutenant l'activité d'identification et de réinhumation des corps.

Ce travail ouvre des pistes sur la formation, ou plutôt l'absence de formation de causes victimaires, soulignant le fait que la perspective de montée en généralité ne découle pas «naturellement» de la condition victimaire, mais dépend d'opportunités politiques et du rapport de force que peuvent exercer les «victimes » dans un contexte spatial et temporel donné. Il serait intéressant de poursuivre cette réflexion dans un cadre comparatif avec d'autres cas de causes autour de la disparition, notamment en Argentine ou au Chili. Ce travail a permis plus localement de réaliser une analyse de long terme de l'impact du travail du Comité pour les personnes disparues de Chypre (CMP) sur la politique locale, sur les relations bilatérales et sur les proches de disparus. Les perspectives à long terme de la cause des victimes de la disparition interrogent. Elle dépend de la capacité du CMP à rendre les corps des disparus. Or, cette ressource de légitimité et de mobilisation s'amenuise au fil des exhumations. Déjà, les cas les plus faciles à déterrer l'ont été et ils ne restent que les cas les plus difficiles, dont il est impossible de garantir qu'ils seront retrouvés. Les horizons ouverts par Together We Can!, notamment la perspective de la judiciarisation des crimes ayant donné lieu à la disparition, par la mise en place d'une commission de vérité par exemple, n'ont pour l'instant pas obtenu d'assise politique. Les perspectives à l'avenir semblent fragiles, alors que la plupart des suspects sont décédés ou arrivent à un âge avancé. Il semble probable aujourd'hui qu'aucun des coupables ne sera jugé et puni. 
90 Politique et sociétés à Chypre aujourd'hui

\section{Bibliographie \\ Monographies}

Ageron Charles-Robert \& Nora Pierre, 1997, Les Lieux de mémoire, Gallimard, Paris, $1642 \mathrm{p}$.

Aktar Ayhan, Kizılyürek Niyazi \& Özkirimli Umut, 2010, Nationalism in the Troubled Triangle Cyprus, Greece and Turkey, Palgrave Macmillan, New York, 267 p.

Bayat Asef, 2010, Life as Politics: How Ordinary People Change the Middle East, Stanford University Press, Stanford, 320 p.

Bryant Rebecca, 2012, Displacement in Cyprus, Consequences of Civil and Military Strife. Report 2. Life Stories: Turkish Cypriot Community, PRIO Cyprus Centre, Nicosia, 64 p.

Cassia Paul Sant, 2004, Bodies of Evidence: Burial, Memory and the Recovery of Missing Persons in Cyprus, Berghahn Books, New York, 304 p.

Copeaux Étienne \& Mauss-Copeaux Claire, 2004, Taksim! Chypre divisée, Aedelsa, Lyon, 240 p.

Fillieule Olivier \& Mathieu Lilian, 2009, Dictionnaire des mouvements sociaux, Presses de Sciences Po, Paris, $651 \mathrm{p}$.

Halbwachs Maurice, 1925, Les Cadres sociaux de la mémoire, version numérisée, Université de Chicoutimi, Chicoutimi.

Lagroye Jacques, 2003, La Politisation, Belin, Paris, 576 p.

LAvabre Marie-Claire, 1994, Le Fil rouge. Sociologie de la mémoire communiste, Presses de Sciences Po, Paris, 320 p.

Lecombe Delphine, 2014, Nous sommes tous en faveur des victimes : la diffusion de la justice transitionnelle en Colombie, Institut universitaire Varenne, Bayonne, $418 \mathrm{p}$. 
Lefranc Sandrine \& Mathieu Lilian, 2009, Mobilisations de victimes, Presses universitaires de Rennes, Rennes, 220 p.

Neveu Érik, 2014, Sociologie des mouvements sociaux, $6^{\mathrm{e}}$ édition, La Découverte, Paris, 128 p.

Ricoeur Paul, 2000, La Mémoire, l'histoire, l'oubli, Éditions du Seuil, Paris, $675 \mathrm{p}$.

\section{Articles et participations à des ouvrages collectifs}

Bozkurt Umut, 2012, “The Potential for Reconciliation, Forgiveness, and Social Transformation" in Trimikliniotis Nicos \& Bozkurt Umut (eds), Beyond a Divided Cyprus: A State and Society in Transformation, Palgrave Macmillan, New York, pp. 201-216.

Branche Raphaëlle, Picaudou Nadine \& Vermeren Pierre, 2014, «Introduction » in Autour des morts de guerre Maghreb - Moyen-Orient, Publications de la Sorbonne, Paris, p. 5-19.

Diamond Larry, 1994, "Rethinking Civil Society: Toward Democratic Consolidation" in Journal of Democracy, $\mathrm{n}^{\circ}$ 3, vol. 5, pp. 4-17, DOI : 10.1353/ jod.1994.0041.

Diaz Paola \& Gutiérrez Ruiz Carolina, 2009, «Les détenus disparus, victimes ou résistants? Les catégories dans la mobilisation de l'Association chilienne des familles de détenus disparus » in Lefranc Sandrine \& Mathieu Lilian (dir.), Mobilisations de victimes, Presses universitaires de Rennes, Rennes, p. 129-144.

Kyriakou Nikolas, 2011, "Enforced Disappearances in Cyprus: Problems and Prospects of the Case Law of the European Court of Human Rights" in European Human Rights Law Review, n 12, vol. 1, pp. 190-199.

Lamarche Karine, 2011, «S'engager “corps et âme” 》 in Cultures \& conflits, $\mathrm{n}^{\circ} 81-82$, p. $125-150$.

Mathieu Lilian, 2007, «L'espace des mouvements sociaux » in Politix, $\mathrm{n}^{\circ} 77 / 1$, p. $131-151$. 


\section{CAHIERS BALKANIQUES}

92 Politique et sociétés à Chypre aujourd'hui

PÉchu Cécile, 2001, «Les générations militantes à droit au logement » in Revue française de science politique, vol. 51, p. 73-103.

Roussel Violaine, 2009, «Les “victimes” : label ou groupe mobilisé ? Éléments de discussion des effets sociaux de la catégorisation » in Lefranc Sandrine \& Mathieu Lilian (dir.), Mobilisations de victimes, Presses universitaires de Rennes, Rennes, p. 101-112.

SiméAnt Johanna, 2003, «Un humanitaire apolitique ? » in LAGroye Jacques (dir.), La Politisation, Belin, Paris, p. 163-196.

Smaoui Sélim, 2014, « Sortir du conflit ou asseoir la lutte ? » in Revue française de science politique, $\mathrm{n}^{\circ}$ 3, vol. 64, p. 435-458.

Smaoui Sélim, 2015, « Fouiller... Militer » in Politix, $\mathrm{n}^{\circ} 110$, p. 35-62.

UludAĞ Sevgül, 7 juillet 2013, http://sevgululudag.blogspot.fr/2013/07/thetwo-missing-brothers-from-dochni.html (consulté le 5/6/16).

\section{Entretiens et documents officiels}

A, Ahmet, et Hüseyin G. 2016. 61 ans et 72 ans, Directeur et membre de l'Association pour la Valorisation des Martyrs de Murataga Sandallar et Atlilar. TC.

« Entretien Huseyin Akansoy.pdf ».s. d.

G, Ayşe, 2016, 56 ans, mère au foyer, père enlevé en 1963.

Hatay Mete, 2016, Politiste, PRIO Cyprus.

«Loi 7/1974, Aides aux familles des Martyrs et des Vétérans de Guerre ». s. d.

A, E, I Palabiyik, 2016, Famille, mari/père disparu en 1963.

B, Sevilay, 2016, 61 ans, vendeuse de tapisserie à la retraite, parents enlevés en 1964, retrouvés en 2012, Turc-Chypriote.

Uluboy Ziliha. 2016, Psychologue TC du CMP depuis 2007. 
Uluda ̆ Sevgül, 2016, 58 ans, journaliste et activiste, membre de Together We Can!

UludaĞ Sevgül, 2004, « Yeraltı Notları, Sevgül Uludağ », 8 décembre 2004.

US Diplomacy, Confidential, 2007, “Missing Persons Committee optimistic on return of remains, but jittery about possible backlash”, Public Library of US Diplomacy.

Von König Florian, 2016, Secrétaire Permanent du CMP, associé au membre de l'ONU, en poste sur Chypre depuis 2008.

Résumé: le présent article montre comment s'est constituée la «cause » des proches de disparus au sein de la communauté turco-chypriote; il étudie la manière dont cette disparition est ou n'est pas vue comme un problème public, une source d'engagement militant et une souffrance par différents groupes sociaux. Il montre ensuite que le rythme de la politique communautaire turco-chypriote et des négociations sur la réunification de l'île influence le développement de cette cause. Après avoir montré comment le référendum de 2004 constitue un tournant, nous rendrons compte du travail des divers groupes d'acteurs qui s'engagent actuellement dans cette cause. L'analyse s'appuie sur une série d'entretiens semi-directifs auprès de proches de disparus, de membres du Comité pour les Personnes disparues de Chypre et de membres de l'association "Together We Can!" à Chypre, entre janvier et mars 2016, et d'un travail d'exploitation d'archives et de sources secondaires.

Mots-clefs : disparus, communauté chypriote turque, post-conflit, politisation, mouvement bicommunautaire, Chypre, Chypre Nord, référendum sur le plan Annan, années 1963-2016, sociologie des causes victimaires, sociologie des organisations, histoire sociale et politique

Abstract: The following paper explains how was created the cause of missing people's relatives in the Turkish-Cypriot community. The main entrance of the paper shows the way disappearance is or is not seen as a public issue, a source of activist engagement and a specific grief by different social groups. This work shows how the rhythm of the Turkish-Cypriot communal politics and the international negotiations on the reunification of the island influence the growth of this cause. We display how the 2004 referendum represents a turning point. Then, we present the work of the different groups engaged in the cause of the missing people's relatives. The paper relies 
on a series of semi-directive interviews with relatives of missing people, members of the Committee for Missing People in Cyprus and members of the non-governmental organization “Together We Can!", in Cyprus, between January and March 2016. The data collected have been checked and completed using archives and secondary sources.

Keywords: disappearance, Turkish-Cypriot community, post-conflict, politicization, bicommunal movement, Cyprus, Northern Cyprus, referendum on the Annan plan, 1963-2016, sociology of victimary causes, sociology of organizations, social and political history

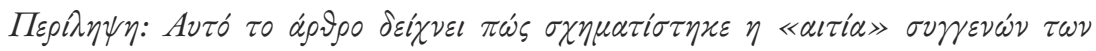

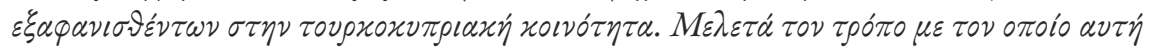

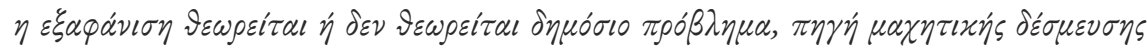

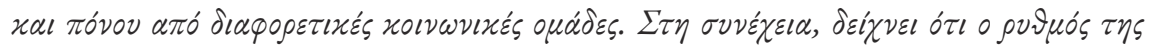

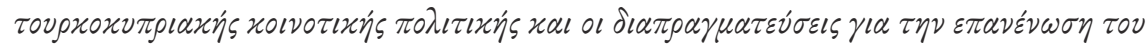

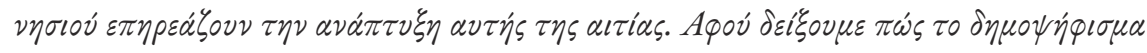

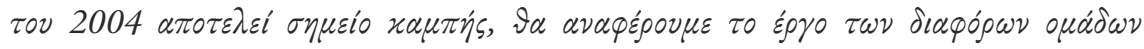

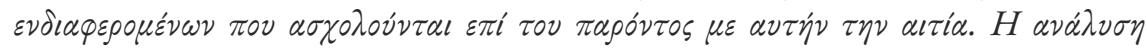

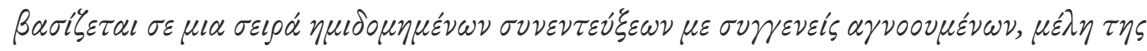

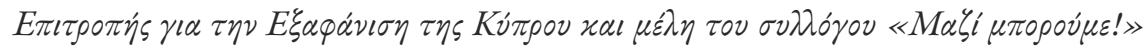

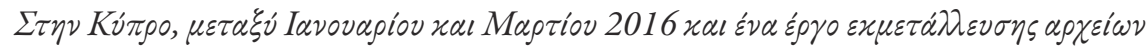

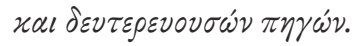

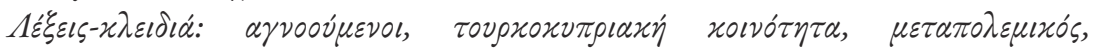

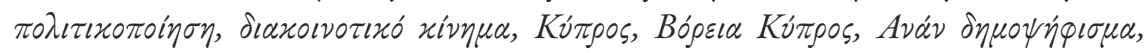

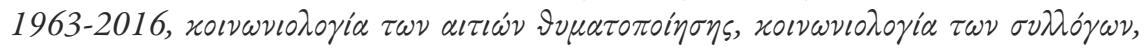

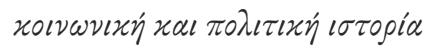

Anabtar kelimeler: kayıp problemi, Kıbrıs Türk toplumu, çatışma sonrası, siyasallaşma, iki toplumlu hareket, Kibrıs, Kuzey Kibrıs, Annan planı referandumu, 1963-2016, mağdur nedenleri sosyolojisi, örgüt sosyolojisi, sosyal ve politik tarih

Клучни зборови: заедница на кипарските Турци, пост-конфликт, политизачија, бикомунистичко движење, Кипар, Северна Кипар, референдум за план на Анан, 1963 година 2016, сочиологија на причините на жртвите, сочиологија на организации, социјална и политичка историја 